\title{
Optimal Swarm Formation for Odor Plume Finding
}

\author{
Ali Marjovi and Lino Marques
}

\begin{abstract}
This paper presents an analytical approach to the problem of odor plume finding by a network of swarm robotic gas sensors and finds an optimal configuration for them, given a set of assumptions. Considering cross-wind movement for the swarm, we found that the best spatial formation of robots in finding odor plumes is diagonal line configuration with equal distance between each pair of neighboring robots. We show that the distance between neighboring pairs in the line topology depends mainly on the wind speed and the environmental conditions, whereas, the number of robots and the swarm's crosswind movement distance do not show significant impact on optimal configurations. These solutions were analyzed and verified by simulations and experimentally validated in a reduced scale realistic environment using a set of mobile robots.
\end{abstract}

Index Terms-Odor Plume Finding, Olfactory Search, Swarm Robotics Formation, Gas Sensor Coverage.

\section{INTRODUCTION}

Searching for olfactory targets with mobile robots has received much attention in the recent years. This problem finds applications in environmental monitoring [1], chemical leak detection [2], pollution monitoring [3], inspection of landfills [4], and search and rescue operations [5]. Some of these tasks are done in scenarios extremely dangerous for humans, being desirable to use robots instead.

The effort to design and develop efficient robotic olfactory search strategies faces the problem of understanding how the odor molecules disperse through the environment under naturally turbulent flow. Odor patches released by an odor source are mainly transported by the airflow, forming an odor plume. As the plume travels away from the source, it becomes more diluted due to molecular diffusion and turbulence that mixes the odor molecules with clean air [6]. Molecular diffusion is a slow process whose effect on the plume shape and the internal concentration can be neglected. The dispersion of odor molecules is dominated by flow turbulence in ventilated indoor or in outdoor environments. The odor molecules move downwind due to mean flow velocity $\vec{U}$ while their net motion is almost random, due to small scale turbulence curls. As the flow carries patches of odor, the average concentration within a patch decreases away from the source, and the average time between successive patches increases. The instantaneous odor concentration strongly fluctuates intermittently with peaks up to three orders of magnitude above the average concentration value [7] (Fig. 1 presents the nature of an odor plume at different scales).

This work was entirely carried out in the Institute of Systems and Robotics, University of Coimbra, Portugal and was partially supported by the European project TIRAMISU under grant FP7/SEC/284747.

Ali Marjovi is currently with the Distributed Intelligent Systems and Algorithms Laboratory at EPFL, Switzerland. e-mail: ali.marjovi@epfl.ch

Lino Marques is with the Institute of Systems and Robotics, University of Coimbra, Portugal. e-mail: lino@isr.uc.pt

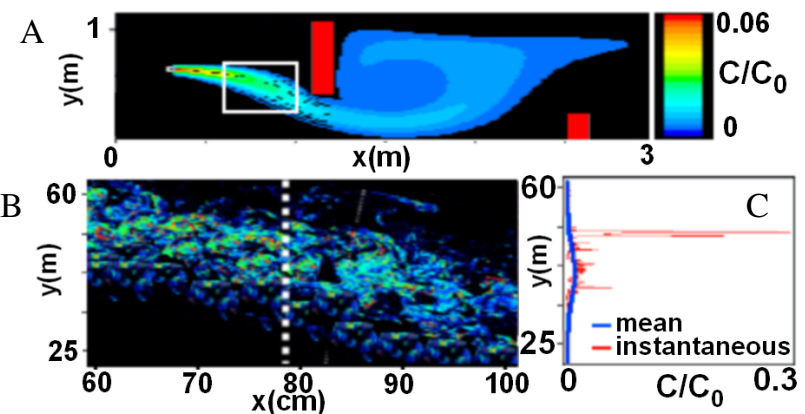

Fig. 1. Odor plume structure in an environment with obstacles. A. mean structure, B. instantaneous structure, and C. instantaneous slice. Picture is adapted from [7]

Under these circumstances, a chemical sensor located far enough downwind of the odor source can only detect the odor peaks and will measure no odor concentration most of the time. The probability of encountering an odor patch at any given point is determined by the relative location of the sensor to the odor source, the statistics of the flow and the shape of the environment and the obstacles [8], [9]. "The mean velocity (and direction) of the wind is set by the largescale atmospheric conditions and hence stays unchanged for periods of time long compared with the time-scale of odor fluctuations" [10].

Finding an odor plume, i.e., searching the environment randomly or systematically in order to find odor clues is the final goal of this study. This is the first phase in search for odor sources [11]. The second phase is plume tracking, that is, following the plume towards the source, and the final step is source declaration, that is, accurately localizing the source [12]. Kowadlo and Russel [13] and Ishida et al. [14] have provided detailed literature reviews about this subject.

Most of the works concerning olfactory search have focused on odor plume tracking [15]-[20] and mapping [21][23], whereas plume finding has received little attention. Bio-inspired [16], [17], concentration gradient climbing (chemotaxis) and up-wind directed search (anemotaxis [19], [24], [25]) are the most common approaches to track odor plumes by mobile robots. Several other methods have been proposed for plume tracking using swarm robotic concepts, namely, biasing expansion swarm approaches (BESA) [26], biased random walk (BRW), evolutionary strategies [27], particle swarm optimization (PSO) [28]-[30], glowworm swarm optimization (GSO) [31], gradient climbing techniques, swarm spiral surge [32], physics-based swarming approach [33], and attraction/repulsion forces [15]. Most of these studies (e.g. [15]-[18], [32]) assume that the robots start their search within or very near the plume.

Since in "odor plume finding" the robots are outside of 
the plume, odor concentration and variance are both zero. Therefore, this problem is usually addressed through general exploration methods [34], mapping [22], [23], or coverage techniques namely zig-zag sweeping, casting, biased random walks [35], lévy-taxis [36], and spiral movements [37], which are also used for other spatial search tasks and are not specifically and efficiently designed for odor plume finding.

Since the search space may be much larger than the active area of an odor source, a mobile sensor network can be advantageous in odor plume finding tasks, in comparison to a single robot that can measure only the odor concentration on its own place. Using multiple sensing nodes spread throughout the environment improves the detection process, increasing the probability of finding an odor plume in a given time. A swarm of robots can establish a dynamic mobile sensor network and move in the area of interest to find the plume. To efficiently address the problem of odor plume finding by a swarm of robots, one should answer the following questions:

1) What is the best movement strategy for the swarm in odor plume searching?

2) What is the best spatial formation for the swarm robots in searching for an odor plume?

These questions seem dependent upon each other and probably the best spatial formation for a particular movement strategy is not the best for another movement strategy. Regarding the first question, it is generally accepted and analytically proved that cross-wind movement is the best strategy to acquire the maximum of information in searching for odor plumes in an unknown environment under stable airflow [38]. Assuming a swarm of robots whose global movement is across the wind, we can study its best spatial formation and answer the second mentioned question. There are a few works in sensor networks literature which study various challenges of using gas sensors (e.g., deployment [39]) and their applications (e.g., environmental monitoring [40]). The most relevant work to this paper is the one in [41] that we analytically found the optimum deployment topology of multiple gas sensors for detecting odor plumes. However, none of these works have taken the mobility of the sensors into account. This mobility implies that coverage area of the sensor network dynamically changes while the robots navigate in the environment.

To state the problem, consider a swarm of $N$ robots that are able to communicate with each other over a distance $\Delta_{d}$ and are equipped with olfactory sensors for sensing the odor concentration $\bar{C}$ and airflow speed $\vec{U}$. There is no central controller for the system, so the robots act independently. The swarm moves cross-wind to find any possible odor plume in an area. The problem is: "what is the best spatial formation strategy for the swarm in search for an odor plume?"

The main novelty of this paper is to present an analytical method to find the optimal spatial formation of swarm robots in plume finding considering cross-wind global movements for the swarm (described in section II). Defining single and multiple mobile gas sensors coverage in different environmental conditions are among the contributions of this paper. Moreover, we present and design a set of wind-biased virtual attractive/repulsive control forces for the swarm robots such that their emergent behavior converges to the optimal
TABLE I

STANDARD DEVIATIONS FOR AN URBAN ENVIRONMENT IN VARIOUS ENVIRONMENTAL CONDITIONS [44].

\begin{tabular}{|l|c|c|}
\hline Env. & $\sigma_{y}(x)$ & $\sigma_{z}(x)$ \\
\hline A-B & $0.32 x(1+0.0004 x)^{-0.5}$ & $0.24 x(1+0.001 x)^{0.5}$ \\
\hline C & $0.22 x(1+0.0004 x)^{-0.5}$ & $0.20 \mathrm{x}$ \\
\hline D & $0.16 x(1+0.0004 x)^{-0.5}$ & $0.14 x(1+0.0003 x)^{-0.5}$ \\
\hline E-F & $0.11 x(1+0.0004 x)^{-0.5}$ & $0.08 x(1+0.0015 x)^{-0.5}$ \\
\hline
\end{tabular}

formations (explained in section III). None of the previous control systems in olfactory robotics community has ever biased the virtual forces by the wind effect. The optimization results were validated and evaluated by experiments in small scale realistic environments (in section IV).

\section{Optimal Coverage with Mobile Gas Sensors}

This section finds the best formation of mobile robotic gas sensors to maximize their sensing coverage area during their crosswind movements.

\section{A. Odor Dispersion Model}

Probability density function of odor dispersion in a turbulent medium is represented by the pseudo-Gaussian model for odor distribution in average-term exposure [6], [8], [9]. The pseudoGaussian plume models yield results that match experimental results reasonably well [42]. If an odor source is located in position $(0,0,0)$, its release rate is $Q$ and the average wind speed is $\bar{U}$ towards $\mathrm{x}$-axis (Fig. 2), then, the mean concentration of odor in position $(x, y, z)$ is given by the following probability density function:

$$
\bar{C}(x, y, z)=\frac{Q}{2 \pi \bar{U} \sigma_{y}(x) \sigma_{z}(x)} \exp \left\{\frac{-y^{2}}{2 \sigma_{y}^{2}(x)}+\frac{-z^{2}}{2 \sigma_{z}^{2}(x)}\right\}
$$

where $\mathrm{x}, \mathrm{y}$, and $\mathrm{z}$ (here and throughout this article) denote the downwind, crosswind, and vertical coordinates relative to the odor source, with $\mathrm{x}$ positive along mean wind direction $\vec{U}$.

Temperature difference in various heights causes an airflow in the vertical direction (z-axis) and makes the plume to rise up/down, yielding to different odor dispersion models. Based on the temperature gradient at the plume height $(\delta T / \delta z)$, the environmental conditions are classified to six categories $\mathrm{A}$ to F (A: neutral, B: slightly stable, C: stable, D: isothermal, E: moderate inversion, $\mathrm{F}$ : strong inversion) [43]. In neutral (A) conditions, the temperature difference between various heights in the environment is close to zero while in strong inversion (F) conditions, the temperature difference in various heights is significant.

The standard deviations $\sigma_{y}(x)$ and $\sigma_{z}(x)$ model the horizontal and vertical dispersion of the plume. These standard deviations are not constant. It was found experimentally by Briggs et al. [44] that both parameters are functions of the downwind distance from the source $(x)$ according to the environmental conditions (A to F), as expressed in Table I. Fig. 2 shows two examples of the mean concentration in two set of environmental conditions, based on equation (1) and Table I. 

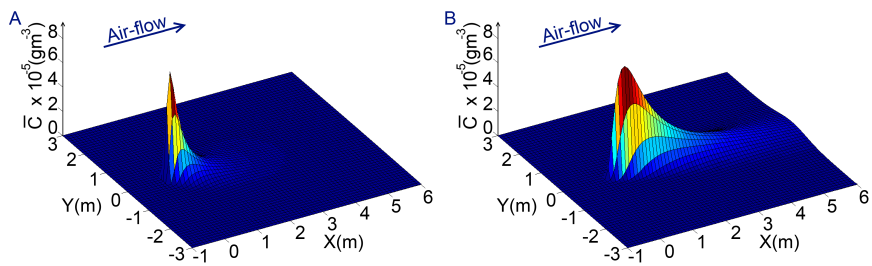

Fig. 2. The mean concentration in the $2 \mathrm{D}$ plane of $z=0.1 \mathrm{~m}$. Source location: $(0,0,0)$, release rate $=0.01 \mathrm{~g} / \mathrm{s}$ and $\bar{U}=1 \mathrm{~m} / \mathrm{s}$ in A-B environmental conditions (A) and E-F environmental conditions (B), (see Table I).
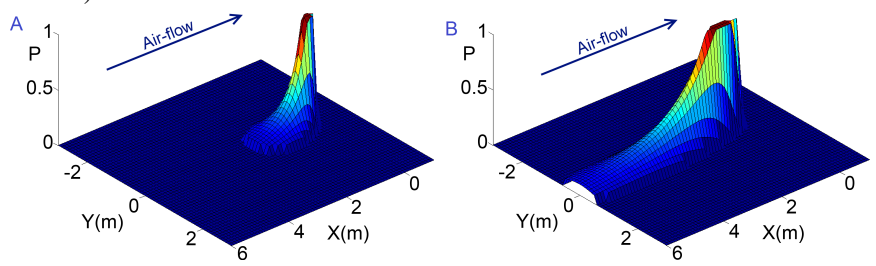

Fig. 3. The probability of detecting odor patches by a sensor at $(0,0)$ if the odor source is located in various points in the plane, when $k=10^{5}$, source release rate $=0.01 \mathrm{~g} / \mathrm{s}$ and $\bar{U}=0.5 \mathrm{~m} / \mathrm{s}$ in A-B environmental conditions (A) and E-F environmental conditions (B).

\section{B. Gas Sensor Area Coverage}

Most gas sensors show pseudo-linear responses to gas concentrations [45]. Considering the odor dispersion model in (1) at a fixed height $(\mathrm{z}=$ constant $=$ source height $)$ this $3 \mathrm{D}$ phenomena can be treated as a 2D problem (similar to [10] and [46]). If an odor source is located at $O\left(x_{0}, y_{0}\right)$, the conditional probability of detecting odor patches by a stationary gas sensor located in position $(x, y)$ is given by:

$$
P\left(D_{x y} \mid O_{x_{0} y_{0}}\right)=\frac{k Q}{2 \pi \bar{U} \sigma_{y}(x) \sigma_{z}(x)} \exp \left\{\frac{-\left(y-y_{0}\right)^{2}}{2 \sigma_{y}^{2}(x)}\right\}
$$

where $k$ is the sensitivity parameter of a gas sensor to the odor concentration. In other words, if a sensor is located in position $(x, y)$, its probability of detecting an odor patch released from a source located in position $\left(x_{0}, y_{0}\right)$ is given by $P\left(D_{x y} \mid O_{x_{0} y_{0}}\right)$ in (2). Equation (2) defines that the higher the odor concentration, the higher the probability of being detected by a sensor. It should be mentioned that the environment in this model (and throughout this paper) is presented by uniform grid maps, so any Cartesian $(x, y)$ denotes a grid cell with center at $(x, y)$. Another point is that since this equation presents a probability function, its result is truncated to $[0,1]$. Although this equation has been simplified by considering $z=$ constant $=z_{0}$, standard deviations of vertical direction $\left(\sigma_{z}(x)\right)$ exists and plays a significant role in this probability function. From (2), if there is a gas sensor at a given position $(x, y)$, where $\left(y-y_{0}\right)>>\sigma_{y}(x)$, its probability of detecting an odor patch is very small. Fig. 3 is an example that presents the distribution of this probability when a sensor is located at $(0,0), \bar{U}$ is $1 \mathrm{~m} / \mathrm{s}$, and the environmental condition is in moderate/strong inversion conditions (E-F type). As it is shown in Fig. 2 and Fig. 3, the odor plume emitted from a source shapes towards the airflow direction, whereas, the probability of detection of a gas sensor shapes in the opposite direction of the airflow.

Given $N$ independent sensors $s_{i}$ located at $\left(x_{i}, y_{i}\right)$, $i=1 . . N$, we compute the total probability $P\left(D_{\left(x_{i}, y_{i}\right)_{i=1}^{N}} \mid O_{x_{j} y_{j}}\right)$, due to the combined efforts of all sensors, of detecting odor patches released from a source in $O\left(x_{j}, y_{j}\right)$. This probability is one minus the probability that all sensors fail to detect:

$$
P\left(D_{\left(x_{i}, y_{i}\right)_{i=1}^{N}} \mid O_{x_{j} y_{j}}\right)=1-\prod_{i=1}^{N}\left\{1-P\left(D_{x_{i} y_{i}} \mid O_{x_{j} y_{j}}\right)\right\}
$$

In other words, if an odor source is located at $O\left(x_{j}, y_{j}\right)$, the probability that at least one sensor (from $N$ applied sensors) detects odor patches is given by $P\left(D_{\left(x_{i}, y_{i}\right)_{i=1}^{N}} \mid O_{x_{j} y_{j}}\right)$ in (3).

The probability functions (2) and (3) inherently define probabilistic coverage area for gas sensors. To obtain the area covered by the gas sensors, a sensitivity threshold $S_{t h}$ for the probability of odor patch detection should be considered. This is based on the fact that most of the gas sensors show a sensitivity threshold i.e. bellow a certain level of odor concentration, the sensors do not detect any odor patch. Thus, this paper defines the binary coverage of a gas sensor to a point of interest as following:

Definition 1 (Single Gas Sensor Binary Coverage). Given a standstill sensor $s_{i}$ in position $\left(x_{i}, y_{i}\right)$ and a point of interest $p_{j}=\left(x_{j}, y_{j}\right)$ the coverage of the sensor $s_{i}$ to the point $p_{j}$ is defined as:

$$
C_{s}\left[s_{i}\left(x_{i}, y_{i}\right), p_{j}\right]= \begin{cases}1, & P\left(D_{x_{i} y_{i}} \mid O_{x_{j} y_{j}}\right)>S_{t h} \\ 0, & \text { Otherwise }\end{cases}
$$

where $P\left(D_{x_{i} y_{i}} \mid O_{x_{j} y_{j}}\right)$ is given by (2).

Fig. 4 presents the coverage area of one standstill gas sensor when the wind is towards left direction. Despite most of the other types of sensors (e.g. acoustic, thermal, vision) whose coverage is either circular or directional sectors towards sensor's heading, the coverage area of gas sensors is ellipsoid shape biased towards the upwind direction.

Assuming crosswind movement for a robotic gas sensor, the binary coverage of the mobile sensor $s_{i}$ to the point $p_{j}$ during its crosswind traveling is defined:

Definition 2 (Single Mobile Gas Sensor Binary Coverage in Crosswind Movement). Given a sensor $s_{i}$, moving crosswind from position $\left(x_{i}, y_{i}\right)$ to position $\left(x_{i}, y_{i}+L\right)$, and a point of interest $p_{j}=\left(x_{j}, y_{j}\right)$, the coverage of the sensor $s_{i}$ to the point $p_{j}$ is defined as:

$$
C_{m}\left[s_{i}, L, p_{j}\right]= \begin{cases}1, & \sum_{l=0}^{L} C_{s}\left[s_{i}\left(x_{i}, y_{i}+l\right), p_{j}\right]>0 \\ 0, & \text { Otherwise }\end{cases}
$$

where $L$ is the number of unit steps (grids) that the mobile sensor travels crosswind in the environment.

This definition implies that a mobile sensor covers a given point $p$ if at least in one location across its trajectory it can detect odor patches released from a possible odor source located in $p$.

With the knowledge of the coverage between sensor $s_{i}$ and all points of interest, the overall coverage can be defined by aggregation. If there are $m$ points of interest, then the total coverage by a sensor $s_{i}$ while traveling crosswind is: 


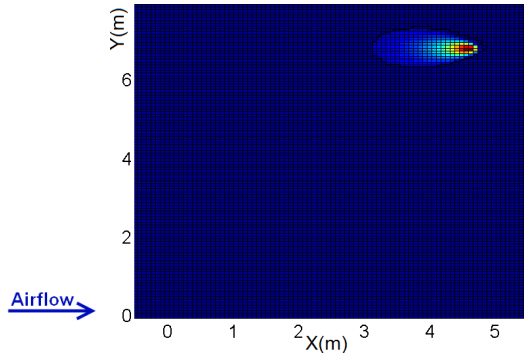

Fig. 4. Probabilistic coverage area of a standstill gas sensor when $S_{t h}=0.3$ $k=10^{5}, Q=0.01 \mathrm{~g} / \mathrm{s}$, and $U=0.2 \mathrm{~m} / \mathrm{s}$ in an environment in Neutral/slightly stable (A-B) conditions based on equations (2) and (4). The direction of airflow in this figure (and throughout this paper) is from left to right.
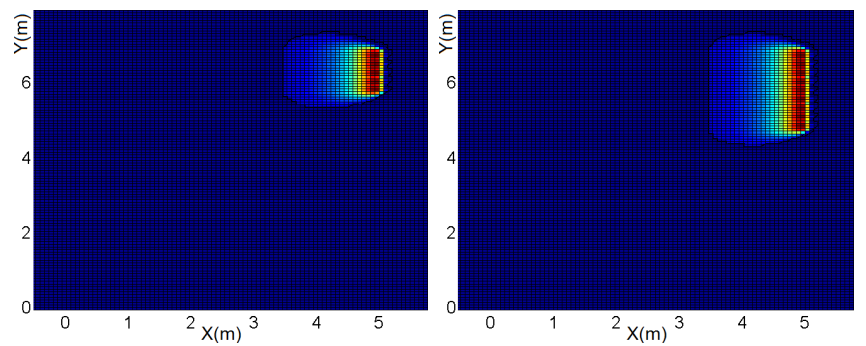

Fig. 5. Coverage area of a mobile gas sensor traveling for $1 m$ (left), and $2 m$ (right). The environmental conditions are the same as in Fig. 4
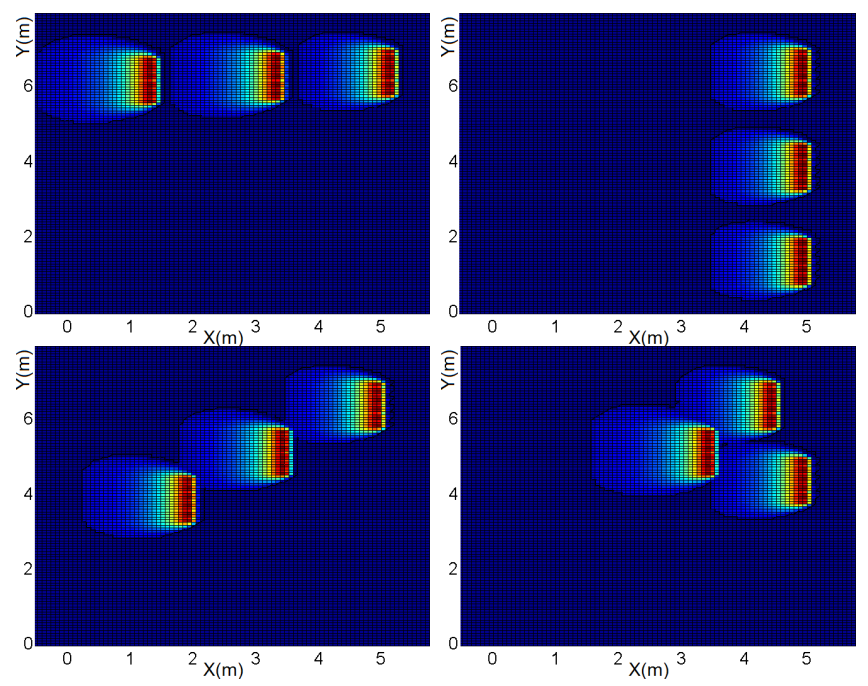

Fig. 6. Coverage area of three mobile gas sensors traveling for $1 \mathrm{~m}$ with different spatial formations.

Definition 3 (Overall Coverage by a Mobile Gas Sensor in Crosswind Movement). The overall coverage by a mobile sensor $s_{i}$ over a region with $m$ points of interest in $R^{2}$ while traveling for $L$ unit steps is given by:

$$
\operatorname{cover}\left[s_{i}, L\right]=\sum_{j=1}^{m} C_{m}\left[s_{i}, L, p_{j}\right]
$$

In this paper, $m$ is the total number of grid cells in an environment. Although the coverage is defined in binary form, its nature is still probabilistic. Fig. 5 presents the coverage area of a mobile gas sensor traveling for one and two meters.

Given $N$ sensors $s_{i}$ located at $\left(x_{i}, y_{i}\right), i=1 . . N$, we define their combined coverage to the point $p_{j}$ as:
Definition 4 (N Gas Sensors Binary Coverage). The combined binary coverage of $N$ standstill sensors $s_{i}, i=1 . . N$ on a point $p_{j}$ is defined as:

$$
C_{s}\left[S_{\left(x_{i}, y_{i}\right)_{i=1}^{N}}, p_{j}\right]= \begin{cases}1, & \left.P\left(D_{\left(x_{i}, y_{i}\right)}\right)_{i=1} \mid O_{x_{j} y_{j}}\right)>S_{t h} \\ 0, & \text { Otherwise }\end{cases}
$$

$P\left(D_{\left(x_{i}, y_{i}\right)_{i=1}^{N}} \mid O_{x_{j} y_{j}}\right)$ is given by equation (3) and is the combined probability of detection of odor patches if the odor source is located at $\left(x_{j}, y_{j}\right)$ by $N$ sensors and $S$ denotes the set of $\left(x_{i}, y_{i}\right)$ positions of the sensors.

Assuming crosswind movement for all $N$ robotic gas sensors, the binary coverage of the sensors to the point $p_{j}$ is defined:

Definition 5 (Multiple Mobile Gas Sensors Binary Coverage in Crosswind Movement). Given a set of $N$ sensors $s_{i}, i=1 . . N$, moving crosswind from position $\left(x_{i}, y_{i}\right)$ to position $\left(x_{i}, y_{i}+\right.$ $L)$, and a point of interest $p_{j}=\left(x_{j}, y_{j}\right)$, the coverage of the sensors to the point $p_{j}$ is defined as:

$$
C_{m}\left[S_{\left(x_{i}, y_{i}\right)_{i=1}^{N}}, L, p_{j}\right]= \begin{cases}1, & \sum_{l=0}^{L} C_{s}\left[S_{\left(x_{i}, y_{i}+l\right)_{i=1}^{N}}, p_{j}\right]>0 \\ 0, & \text { Otherwise }\end{cases}
$$

Finally, the overall coverage of $N$ mobile gas sensors traveling crosswind over a region is defined by:

Definition 6 (Overall Coverage by $\mathrm{N}$ Mobile Sensors in Crosswind Movement). The overall coverage by $N$ mobile sensors $s_{i}, i=1 . . N$ traveling crosswind for $L$ unit steps, over a region with $m$ points of interest in $R^{2}$ is given by:

$$
\left.\operatorname{cover}\left[S_{\left(x_{i}, y_{i}\right)_{i=1}^{N}}, L\right]=\sum_{j=1}^{m} C_{m}\left[S_{\left(x_{i}, y_{i}\right)}\right)_{i=1}^{N}, L, p_{j}\right]
$$

This equation implies that the coverage is a function of: sensors' positions $\left(s_{i}, i=1 \ldots N\right)$, source release rate $(Q)$, average wind speed $(\bar{U})$, sensors sensitivity $(K)$, travel distance $(L)$, and distribution standard deviations related to environmental conditions $\left(\sigma_{y}(x)\right.$ and $\left.\sigma_{z}(x)\right)$.

Fig. 6 shows the coverage area of three mobile gas sensors traveling crosswind for one meter holding various spatial formations. It is obvious that in constant environmental conditions the coverage area of $N$ mobile gas sensors depends on their spatial topology.

It must be noted that since the definition of area coverage depends from a detection threshold, the aggregative area coverage depends from the sensors' relative positioning. For $N$ sensors the threshold is applied to the combined probability of all of them in (7), i.e., first the probability of detection for all sensors on a single grid cell is combined and then the threshold is applied. This implies that maximal coverage will be obtained by partial overlapping of individual sensors' coverage area and not with a non-overlapping distribution. Based on the probabilistic coverage definitions in (4) and (7), there exist areas which are covered by the combined ability (probability) of the multiple sensors, but are not covered by any of them if they were alone. Considering this fact, it is a difficult optimization problem to find the locations for the sensors which maximizes the overall coverage. 
TABLE II

PARAMETERS OF OPTIMIZATIONS

\begin{tabular}{|c|c|c|c|}
\hline $\mathrm{N}$ & $\mathrm{L}$ & $\mathrm{U}$ & Env. \\
\hline $2,3,6,10,16$ & $0.5,1,2,4,8$ & $0.1-10$ & A-B, C, D, E-F \\
\hline
\end{tabular}

\section{Optimal sensor deployment}

The aim of this paper is to find the optimal sensors formation in a way that overall coverage is maximized. Thus, we are looking for a series of sensors positions $s_{i}=\left(x_{i}, y_{i}\right)$ such that:

$$
\left\{s_{1}, s_{2}, \ldots, s_{N}\right\}=\arg \max \operatorname{cover}\left[S_{\left(x_{i}, y_{i}\right)_{i=1}^{N}}, L\right]
$$

Optimal sensor formations are the configurations where the coverage area of the sensors is maximized. Therefore maximizing the area of sensor coverage, defined in (9), is used as the criterion of our optimization. We optimize this criterion with various number of sensors and different average wind speeds in four environmental conditions considering various traveling distances $(L)$ for the mobile sensors. Without loss of generality, we assume constant values for the following parameters during the optimizations: $S_{t h}=0.35$, $Q=0.01 \mathrm{~g} / \mathrm{s}, k=10^{5}$ and the environment size is $100 \times 100 \mathrm{~m}^{2}$. These values are close to real world experimental measurements [47].

The Powell's conjugate gradient descent method [48] was used (in Matlab) to optimize this problem, since it does not need the derivative of the function and its convergence is fast even in high dimensional spaces. $N$ sensors on a 2D plane require $2 N$ dimensional search space. For each combination of sensors' position, the coverage area is computed considering mentioned environmental conditions (in Table I) and setup parameters (mentioned in the previous paragraph) and various traveling distances. The solution is a set of sensors' positions for which their coverage area is the largest.

\section{Optimization Results}

The optimal coverage area was measured by different number of sensors from 2 to 16 traveling cross wind for 0.5 , $1,2,4$ and 8 meters, and different wind speeds from 0.1 to $10 \mathrm{~m} / \mathrm{s}$ in the four environmental conditions (see Table II). The topological shape of the sensors in the optimal solutions was analyzed in each case. Fig. 7 shows examples of optimized formation of three mobile sensors while traveling one meter crosswind ( $L=1 \mathrm{~m}$ ) with different values for the wind speed.

Fig. 8 shows another example of optimized configurations for two to five sensors and their maximum coverage area in an urban environment under neutral/slightly stable (A-B) conditions when the wind speed is equal to $1 \mathrm{~m} / \mathrm{s}$ and the swarm's crosswind movement $(L)$ was $1 \mathrm{~m}$. Different values of $U, N$ and $L$ in different environmental conditions result in similar (but not equal) solutions. One interesting point from all of the optimized solutions is that:

Conclusion 1 (Line Topology). The formation of all of the optimal solutions is line configuration, with equal distance between each pair of neighboring sensors.
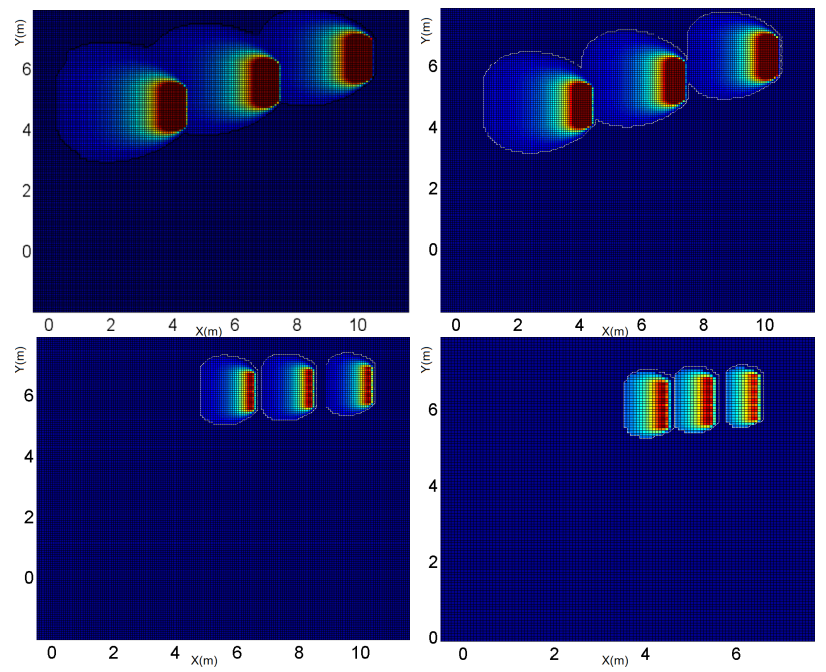

Fig. 7. The optimized formation of 3 mobile gas sensors in an area when the wind speed is $0.2,0.3,1$ and $3 \mathrm{~m} / \mathrm{s}$ in A-B environmental conditions.
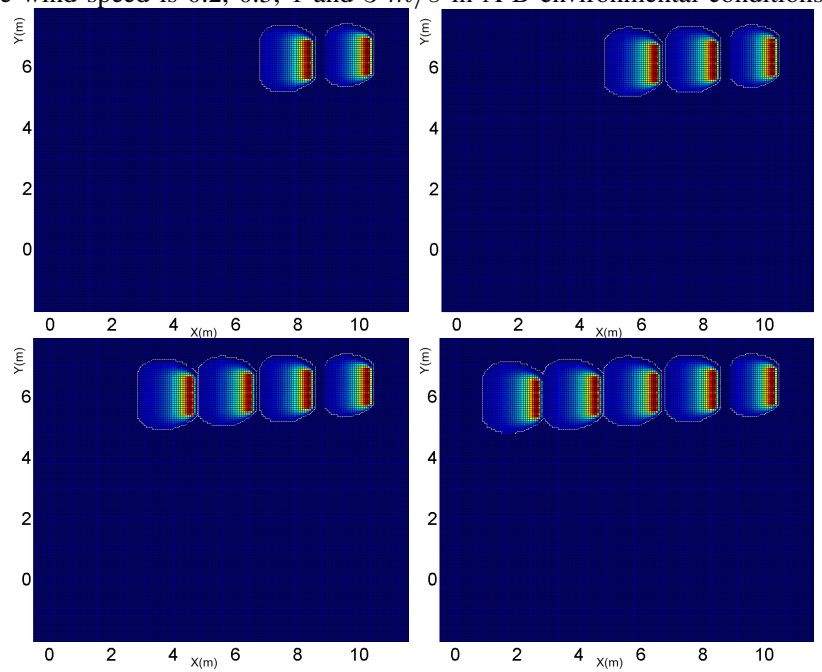

Fig. 8. The optimized formation of 2 to 5 mobile gas sensors in an area when the wind speed is $1 \mathrm{~m} / \mathrm{s}$ in A-B environmental conditions.

The distance between the robots in the optimal formations is called optimal distance. We study the $\mathrm{X}$ and $\mathrm{Y}$ components of the optimal distance in every optimization set (listed in Table II).

Fig. 9 presents some results of the optimizations that show the $\mathrm{X}$ and $\mathrm{Y}$ components of optimal distance between the neighboring sensors in optimized configurations in neutral/slightly stable (A-B) environmental conditions when the number of sensors $(N)$ ranges from 2 to 16 and the swarm's crosswind movement distance $L$ is $1 \mathrm{~m}$. This chart shows that in these conditions, changing $N$ does not affect the inter sensor optimal distance. Although this chart only shows the optimal results in one set of parameters, changing $N$ from 2 to 16 does not show a significant change in the optimization results for other conditions as well. By analyzing these results of the optimizations, it can be seen that, in presence of constant wind speeds, when the number of sensors changes (from 2 to 16), the optimal distance between the sensors changes only for a few centimeters and is almost constant even when the 

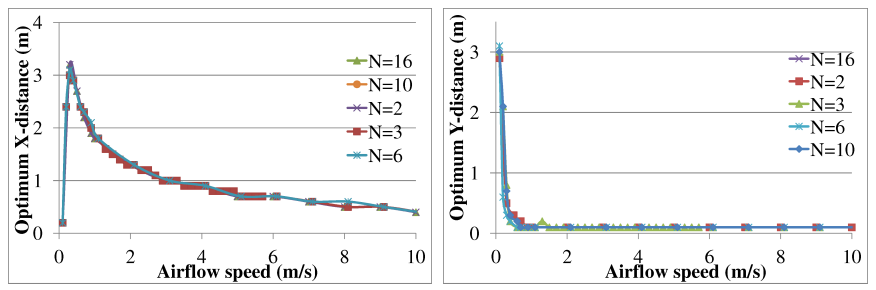

Fig. 9. The $\mathrm{X}$ and $\mathrm{Y}$ components of optimal distance between the neighboring sensors in the optimized configuration in A-B environmental conditions when the number of sensors $(N)$ varies from 2 to 16 and the swarm's crosswind movement distance $(L)$ is $1 \mathrm{~m}$.
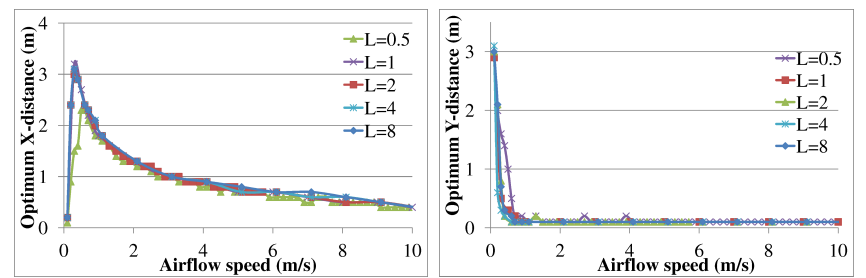

Fig. 10. The $\mathrm{X}$ and $\mathrm{Y}$ components of optimal distance between the neighboring sensors in the optimized configuration in A-B environmental conditions when $N$ is 10 and $L$ varies from 0.5 to $8 \mathrm{~m}$.
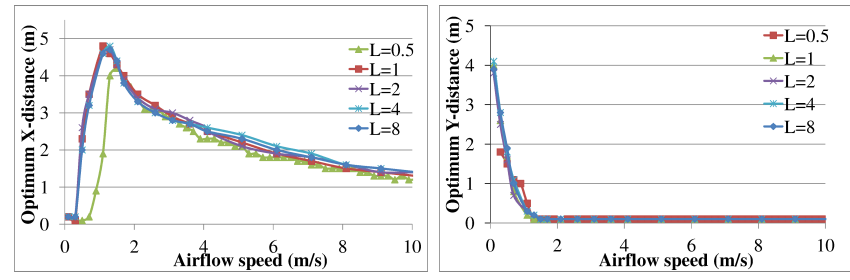

Fig. 11. The $\mathrm{X}$ and $\mathrm{Y}$ components of optimal distance between the neighboring sensors in the optimized configuration in E-F environmental conditions when $N$ is 10 and $L$ varies from 0.5 to $8 \mathrm{~m}$.

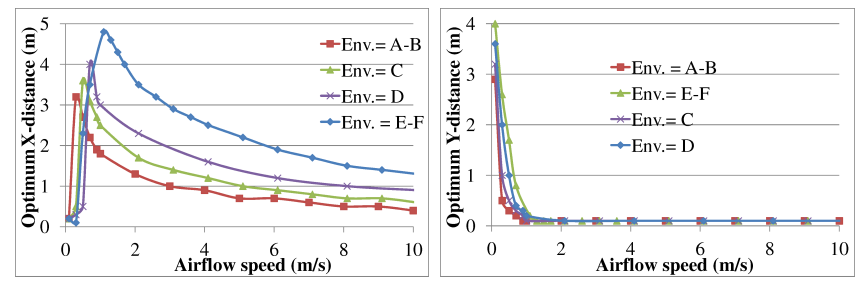

Fig. 12. The optimal distance between the neighboring sensors in the optimized configurations in different environmental conditions. The chart is the average of the results for $N=2$ to 10 and $L=1$ to $8 \mathrm{~m}$.

swarm's movement distance is changed (see the examples in Fig. 8).

Fig. 10 presents another set of optimization results when $N$ is 10 , environmental condition is A-B type, $U$ varies from 0.1 to $10 \mathrm{~m} / \mathrm{s}$ and $L$ has different values from 0.5 to $8 \mathrm{~m}$. This chart shows that different values for L (swarm's crosswind movement distance) results in [almost] the same optimization results. Only when $L$ is very small $(L=0.5 \mathrm{~m})$ (and especially when the wind speed is less than $2 \mathrm{~m} / \mathrm{s}$ ) the optimal distances show different results. For other values of optimization parameters similar results were obtained. Fig. 11 presents the optimization results in another environmental conditions that shows similar conclusions.

By considering the results in Fig. 9, Fig. 10 and Fig. 11 we can conclude that:

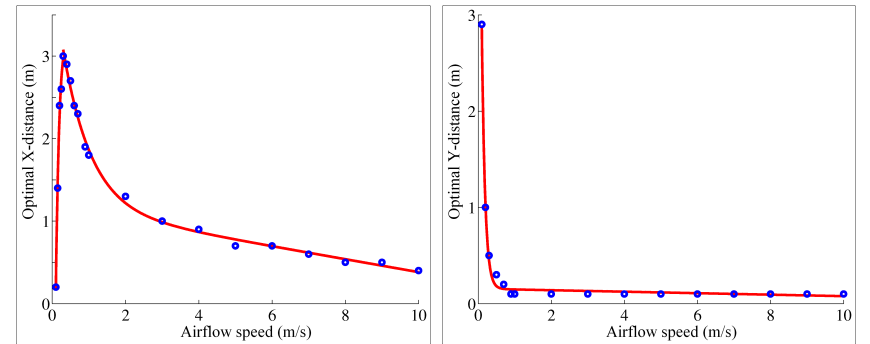

Fig. 13. The average optimal distance between neighboring sensors in A-B environmental conditions and a non-linear regression estimation.

Conclusion 2 (Wind \& Environment Dependent Distance). The distance between neighboring pairs in optimal configurations depends mainly on the wind speed and environmental conditions, whereas, the number of sensors and the swarm's crosswind movement distance do not show significant impact on optimal configurations.

These conclusions are drawn after the results obtained from numerical analysis and are the most significant contributions of this paper.

Fig. 12 shows the effect of environmental conditions on the optimization results. This chart is the average result of optimizations when $N=2$ to 16 and $L=1$ to $8 \mathrm{~m}$. This chart shows that in an environment under neutral/slightly stable conditions the distance (specially X-component) between the neighboring sensors is larger than in an environment under strong inversion conditions. The results in Fig. 12 show that in fixed environmental conditions, when the wind speed is lower than a certain value, the higher wind speed, the larger the optimal X-distance. The certain values (peaks in the graphs) depend on the environmental conditions. It is 0.3 for $\mathrm{A}-\mathrm{B}, 0.5$ for $\mathrm{C}, 0.7$ for $\mathrm{D}$, and 1.1 for $\mathrm{E}-\mathrm{F}$ conditions. However, when the wind speed is more than a certain value, the higher the wind speed is, the smaller the optimal $\mathrm{X}$ distance becomes. Therefore, as the wind speed increases, the $\mathrm{X}$-distance in-between the sensing nodes should decrease in order to maintain optimal coverage. Similarly, when the wind is smaller than a certain value, the higher the wind speed is, the smaller the optimal Y-distance becomes. The Y-distance dramatically decreases and converges to a value close to $0.1 \mathrm{~m}$ when the wind speed is too high. Taking the results shown in Fig. 12 and using a non-linear regression analysis, we obtained the following analytical equation which describes the optimal distance between the sensing nodes as a function of the wind speed in neutral/slightly stable environmental conditions.

$$
\begin{gathered}
D_{x}= \begin{cases}-9.6 e^{-8.8 U}-2.6 U+4.4, & U \leq 0.3 \\
2.8 e^{-1.3 U}-0.07 U+1.1, & U>0.3\end{cases} \\
D_{y}=8.3 e^{-11.1 U}-0.007 U+0.15
\end{gathered}
$$

The red lines in Fig. 13 are the fitted functions (10) and (11) while the scattered blue circles are the results of the optimizations. The mean square error of these regression lines is $4 \times 10^{-3}$. For the other environmental conditions, estimation functions can be calculated in the same way. These functions are later used by mobile robots to estimate the optimal distance based on the wind speed. 
It should be mentioned that the obtained results are valid for specific values of $S_{t h}, k$ and $Q$ defined in section II-C. However, for other sets of assumptions the optimal configuration for the sensors is the same (i.e. a line) and only the values of the optimal distance between the sensors and regression function (10) are changed.

\section{WIND-BIASED POTENTIAL FIELDS}

Conclusions 1 and 2 demand that robots should line-up with equal distances from each other while moving cross-wind, to maximize their probability of finding odor plumes. There is no central controller for swarm robots and the formation topology of the swarm is the emergent result of individual robots movements. Therefore, for the swarm to perform a desired formation topology, each robot should move in the space following a correct and well-defined control strategy. To control the motion of the robots, this section presents a novel method based on the virtual attraction/repulsion forces. Despite previous works on swarm formations, we take the wind direction and the wind speed into account and bias the attraction/repulsion forces by the wind to implement the desired line-up formation. This method is a suitable control strategy for the swarming robots since it does not need a central control node and it is flexible to be modified in order to implement other robotic behaviors (e.g obstacle avoidance). We define a behavior named "diagonal line-up" for the individual robots in order to implement diagonal line formation for the swarm. This behavior defines two types of virtual forces that are applied to the robots; robot-to-robot and robot-to-environment forces.

\section{A. Robot-to-robot forces}

To perform "diagonal line-up" behavior, each robot measures the air-flow direction $\vec{U}$ and assumes this direction as its internal $\mathrm{X}$-axis coordinate system and then it measures the relative distance to its neighboring robots. Afterwards, the robots try to maintain a specific distance in their X-axis from their neighbors and maintain another specific distance with them in their Y-axis. Hence, we define a bounded potential between each pair of neighboring robots $i$ and $j$ at time $t$ :

$$
\begin{gathered}
<\vec{X} \text { axis }>\equiv<\vec{U}> \\
\vec{F}_{d l}^{i j}(t)=\overrightarrow{F x}_{d l}^{i j}(t)+\overrightarrow{F y}_{d l} i j \\
\overrightarrow{F y}_{d l}(t)= \begin{cases}-\mu_{1}\left(\frac{\left\|\vec{Y}_{i j}\right\|-D_{y}}{\left\|p_{i j}(t)\right\|^{2}}\right)\left[\frac{\vec{Y}_{i j}}{\left\|\vec{Y}_{i j}\right\|}\right], & 0<\left\|\vec{Y}_{i j}(t)\right\|<D_{y} \\
-\mu_{2}\left(\frac{\left\|\vec{Y}_{i j}\right\|-D_{y}}{\left\|p_{i j}(t)\right\|^{2}}\right)\left[\frac{\vec{Y}_{i j}}{\left\|\vec{Y}_{i j}\right\|}\right], & D_{y}<\left\|\vec{Y}_{i j}(t)\right\|<D_{r} \\
0, & \left\|\vec{Y}_{i j}(t)\right\|>D_{r}\end{cases} \\
\overrightarrow{F x}_{d l}^{i j}(t)=\left\{\begin{array}{llll}
-\mu_{3}\left(\frac{\vec{x}_{i j}-D_{x}}{\left\|p_{i j}(t)\right\|^{2}}\right), & 0<\left\|\vec{X}_{i j}(t)\right\|<D_{r} & \& & \vec{Y}_{i j} \geq 0 \\
-\mu_{3}\left(\frac{\vec{X}_{i j}+D_{x}}{\left\|p_{i j}(t)\right\|^{2}}\right), & 0<\left\|\vec{X}_{i j}(t)\right\|<D_{r} & \& & \vec{Y}_{i j}<0 \\
0, & \left\|X_{i j}(t)\right\|>D_{r}
\end{array}\right.
\end{gathered}
$$

where

- $\vec{F}_{d l}^{i j}(t)$ is the force applied to robot $i$ by robot $j$ at time t. $\overrightarrow{F x}_{d l}^{i j}(t)$ and $\overrightarrow{F y}_{d l}^{i j}(t)$ are respectively the $x$ and $y$ components of $\vec{F}_{d l}^{i j}(t)$.

- $\left\|p_{i j}(t)\right\|$ is the distance between robots $i$ and $j$.

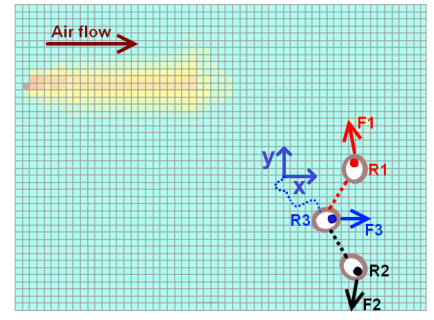

Fig. 14. An example of line-up behavior. Forces applied to the robots based on equations (12-15) when we manually set $D_{x}$ to 0 and $D_{r}=D_{y}=1 \mathrm{~m}$. F1, F2 and F3 demonstrate the total forces applied to R1, R2 and R3 respectively.

- $X_{i j}=x_{i}-x_{j}$ and $Y_{i j}=y_{i}-y_{j}$ where $\left(x_{i}, y_{i}\right)$ is the relative position of robot $i$ and $\left(x_{j}, y_{j}\right)$ denotes the relative position of robot $j$. It is obvious that $\left\|\vec{Y}_{i j}\right\|$ denotes the magnitude and $\left[\frac{\vec{Y}_{i j}}{\left\|\vec{Y}_{i j}\right\|}\right]$ is the direction of the vector $\vec{Y}_{i j}$.

- $\mu_{1}, \mu_{2}$ and $\mu_{3}$ are constant coefficients for tuning acceleration of the robots. $\mu_{1}$ is the Y-component repulsing coefficient and $\mu_{2}$ is the Y-component attracting coefficient while $\mu_{3}$ is the $\mathrm{X}$-component coefficient. It should be mentioned that one single coefficient could be used in the virtual forces. However, we intentionally use 3 different coefficients to make this point that attraction/repulsion coefficients in $\mathrm{x}$ and $\mathrm{y}$ axes can be set to different values. These values affect the acceleration of the robot in each direction.

- \& denotes the logical AND operator.

- $D_{x}$ is a design parameter that specifies the desired Xcomponent distance interval between the neighboring robots. We defined $D_{x}$ using the equation (10) to be equal to the optimization results, considering A-B environmental conditions.

- $D_{y}$ is a design parameter that specifies the desired Ycomponent distance interval between the neighboring robots. We defined $D_{y}$ using the equation (11), considering A-B environmental conditions.

- $D_{r}$ defines the margin of the area that a robot applies forces to the other robots. Logically for line formation $D_{r}$ should be bigger than $\max \left(D_{x}, D_{y}\right)$ and smaller than $2 \times \max \left(D_{x}, D_{y}\right)$. Moreover, $0<\left(D_{x} \& D_{y}\right)<D_{r}<\Delta_{d}$.

The above equations are inspired by the Hooke's law, thus, the forces are similar to the forces in the physical springs. Hence, the robots try to maintain their X-component distance to $D_{x}$ and to maintain a distance of $D_{y}$ in their Y-component distance. The result will be a diagonal line whose slope is $D_{y} / D_{x}$. Fig. 14 shows a simplified example of these forces on three robots. Finally, the total "diagonal line-up" force $\vec{F}_{d l}^{i}(t)$ for robot $i$ is determined as:

$$
\vec{F}_{d l}^{i}(t)=\sum_{j=1 ; j \neq i}^{N} \vec{F}_{d l}^{i j}(t)
$$

It is worth to mention that, although the summation of the forces is over all the other robots $(\mathrm{N})$, only those within the detection range $\left(\Delta_{d}\right)$ of robot $i$ which are closer than $D_{r}$ actually effect the value of $\vec{F}_{d l}^{i}(t)$. 


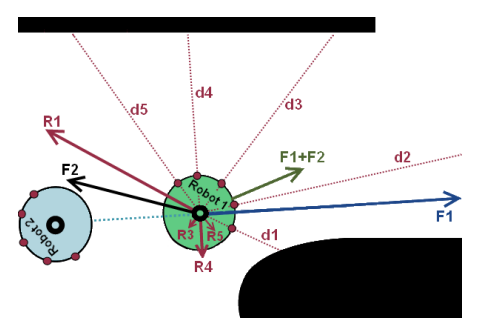

Fig. 15. Obstacle avoidance for a robot with five range sensors. d1 to d5 correspond the distance measured by the sensors. R1 to R5 are the artificial repulsive forces. "F1" represents the artificial robot-to-robot force and "F2" illustrates the summation forces of obstacle avoidance. Vector "F1+F2" shows the total force applied to robot 1 .

\section{B. Robot-to-environment forces}

The low level of autonomous navigation of a robot relies on the ability of the robot to simultaneously achieve its target goal and avoid the obstacles in the environment. To avoid the obstacles, a reactive potential field control method is used. Fig. 15 is an example that shows the virtual potential forces applied to a robot in an environment. Considering $M$ range sensors, we define the forces applied to robot $i$ by its surrounding environment as:

$$
\vec{F}_{o b s}^{i}(t)=\sum_{j=1}^{M} \frac{c_{1}}{\left|d_{i}(j)\right|^{n}} \overrightarrow{\left(V e c_{i j}\right)}
$$

Since $d_{i}(j)$ is simply the distance between robot $i$ and an obstacle that is reported by the range sensor $j$, the force is an inverse function of the distance of the robot to the surrounding obstacles. $\overrightarrow{V e c_{i j}}$ is a predefined vector whose magnitude is set to one and its direction is from sensor $j$ towards the center of robot $i . c_{1}$ is a positive coefficient and $n$ is an even integer.

\section{Swarm movements}

It is desired for the swarm robots to hold the "diagonal line" formation and move cross-wind. Hence, they will sweep and cover the environment towards cross-wind. To do so, each robot should move in the space in the correct direction. Here we set the $\vec{F}_{G}^{i}(t)$, which is a force applied to robots towards their global goal, equal to $\vec{F}_{C r W}^{i}(t)$ that is defined as :

$$
\begin{gathered}
\vec{F}_{C r W}^{i}(t)=\overrightarrow{F x}_{C r W}^{i}(t)+\overrightarrow{F y}_{C r W}^{i}(t) \\
\overrightarrow{F x}_{C r W}^{i}(t)=0 \\
\overrightarrow{F y}_{C r W}^{i}(t)== \begin{cases}0, & \left\|\vec{F}_{d l}^{i}(t)\right\|>F s_{t h} \\
F_{\beta}, & \left\|\vec{F}_{d l}^{i}(t)\right\| \leq F s_{t h}\end{cases}
\end{gathered}
$$

where $\overrightarrow{F x}_{C r W}^{i}(t)$ and $\overrightarrow{F y}_{C r W}^{i}(t)$ are respectively the $\mathrm{X}$ and Y competent of the defined cross-wind force $\vec{F}_{C r W}^{i}(t) . F s_{t h}$ is a threshold value for the forces applied to a robot and $F_{\beta}$ is a constant predefined force. The above formula checks if $\left\|\vec{F}_{d l}^{i}(t)\right\|$ is bigger than a defined threshold or not. If $\left\|\vec{F}_{d l}^{i}(t)\right\|$ is very small it means that the resultant virtual forces applied to robot $i$ are near zero, i.e, the topology of the robot and its neighbors is in the form of a diagonal line and it is in its steady state. In this case a force $\left(F_{\beta}\right)$ towards Y-axis direction (i.e, cross-wind) is applied to the robot and robot moves towards cross-wind direction.

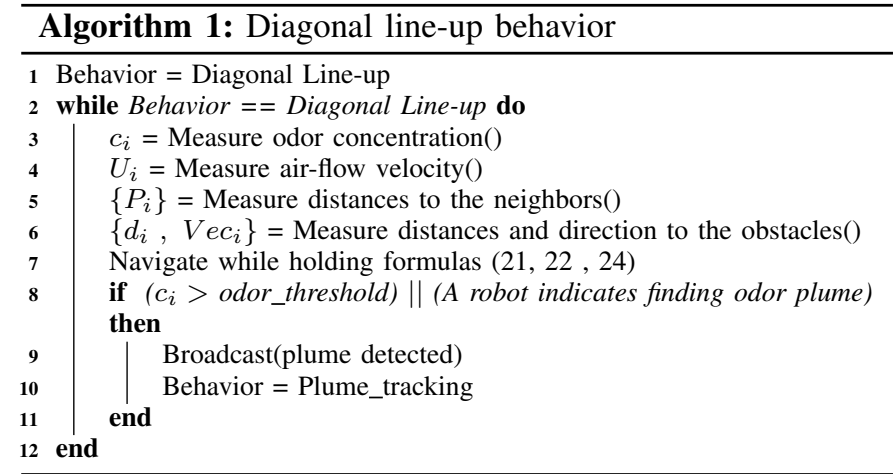

\section{The total force}

The total force applied to a robot in "diagonal line-up" behavior is:

$$
\vec{F}_{s m}^{i}(t)=\vec{F}_{d l}^{i}(t)+\vec{F}_{o b s}^{i}(t)+\vec{F}_{G}^{i}(t)
$$

For a swarm of $N$ individual robots in Euclidean plane, denoting $\theta^{i}(t)$ as the steering angle of robot $i$ at time $t$, the desired direction of motion of robot $i$ is given by:

$$
\theta_{d}^{i}(t)=\arctan \left(\vec{F}_{y}^{i}(t), \vec{F}_{x}^{i}(t)\right)
$$

where $\vec{F}_{y}^{i}(t)$ and $\vec{F}_{x}^{i}(t)$ represent the $x$ and $y$ components of the force $\vec{F}_{s m}^{i}$. Now, a proportional controller is used for the orientation dynamics of the robot:

$$
w_{i}(t)=-\lambda\left(\bmod \left(\left(\theta_{i}(t)-\theta_{d}^{i}(t)\right)+\pi, 2 \pi\right)-\pi\right)
$$

where $\lambda$ is a positive proportional gain and mod is simply the modulo operation which finds the remainder of division of $\left(\left(\theta_{i}(t)-\theta_{d}^{i}(t)\right)+\pi\right)$ by $2 \pi$. Finally, the next velocity of the robot $\vec{v}_{i}(t)$ is calculated based on its last velocity $\vec{v}_{i}(t-\Delta t)$ and the forces applied to it $\vec{F}_{s m}^{i}(t)$;

$$
\vec{v}_{i}(t)=\vec{v}_{i}(t-\Delta t)+\eta \vec{F}_{s m}^{i}(t) \Delta t
$$

while $\eta$ is a constant coefficient multiplied to the acceleration of the robot.

Algorithm 1 presents the pseudo-code that should be run in each robot to perform this behavior. It should be noted that this method always keeps the robots in a diagonal-line formation based on the status of the current airflow conditions. If the direction and the speed of air-flow changes during the experiments, the robots automatically and dynamically change their formation parameters to adapt their topology to the new conditions. Based on (12) the X-axis of the coordinate system of each robot is always aligned with the direction of the airflow. This means that if the direction of the airflow changes over time the direction of the $\mathrm{x}$ and $\mathrm{y}$ axes in coordinate system of the robot also changes and the robots always align their desired topology with the state of the airflow. Moreover, based on (10) and (11), the distance between the neighboring robots dynamically modifies if the airflow speed changes.

The robots maintain diagonal line-up behavior until one (or some) of them gets into an odor plume by sensing odor concentrations higher than a defined threshold. Plume tracking is not in the scope of this paper, however, a robot which gets into the odor plume can perform another behavior to inform 


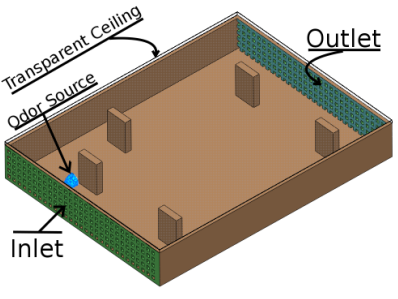

Fig. 16. The model of a testing environment with $4 \times 6 \mathrm{~m}^{2}$ dimensions.
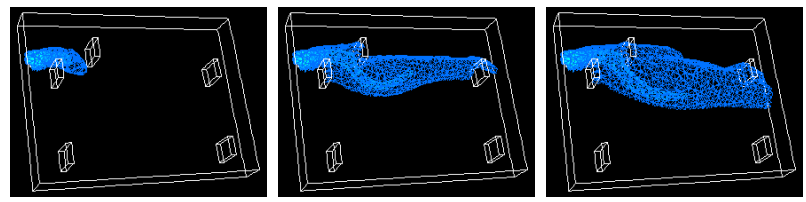

Fig. 17. ANSYS Fluent three dimension simulations; contours of mass fraction of ethanol propagated in the testing environment of Fig. 16.

the other robots to get into the plume and track it. The swarm can cooperatively track the odor plume towards its source (using a method like [15]) and ultimately localize the source.

\section{VALIDATION}

The presented method was validated in both simulations and realistic experiments.

\section{A. Simulations}

The method was tested in several different simulation environments containing obstacles with different number of robots. This section goes to the details of these simulations and presents the results.

1) Testing environment: Models of several testing environments were given to ANSYS Fluent $\mathrm{CFD}^{1}$ software to simulate odor sources and provide odor concentration data. The olfactory data generated by ANSYS Fluent was exported to Matlab to be used in simulations. One of the environments designed for these simulations is depicted in Fig. 16. The dimension of designed arenas for simulations was varied from $4 \times 6 \mathrm{~m}^{2}$ to $30 \times 40 \mathrm{~m}^{2}$. The airflow was ventilated from the inlet side (left) with different speeds from 0.5 to $20 \mathrm{~m} / \mathrm{s}$. In the environments with obstacles, the flow velocity varies in different parts of the arena. Fig. 17 shows several $3 \mathrm{D}$ snapshots of an odor plume propagation during the time in one of the tested scenarios. As shown in the simulation snapshots, the odor propagation is time variant and under turbulent flow. Although the odor plume was simulated in $3 \mathrm{D}$, the robots move in the floor with their gas sensors always at the same height, so, only the odor concentration measured in the 2D plane at the height of the sensors is relevant to the robots' decisions. We extracted the odor concentrations and airflow velocities of 10 centimeters height from the 3-D odor plumes and fed it to the robots in the simulations. Fig. 18 presents some snapshots of an extracted 2D odor plumes in one scenario.

${ }^{1}$ ANSYS Fluent CFD, "FLUENT user's manual" Software Release, vol. 6, 2006.
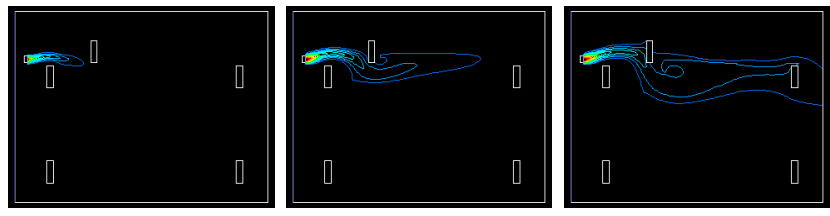

Fig. 18. Extracted 2D odor contours of mass fraction of ethanol propagated in the testing environment of Fig. 17 during the time.
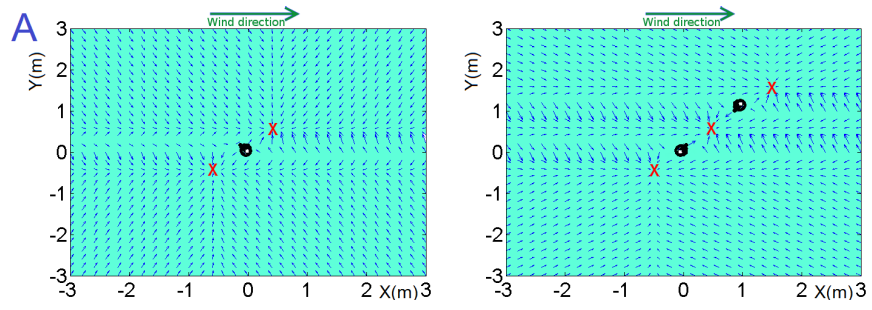

Fig. 19. Virtual forces generated by one (A) and two (B) robots when the wind direction is left to right, and $D_{y}=0.5 \mathrm{~m}, D_{x}=0.5 \mathrm{~m}$. The X marks show the locations that the virtual forces converge to. If another robot is added to this system, it will move to one of the marked places.

2) Robots: Robots were simulated in Matlab as independent entities with no shared variables. The environmental data including odor concentrations, wind speeds and obstacles' locations are shared with the robots such that the robots can measure the odor concentration and air-flow speed of their places. The diameter of the robots in the simulations was set to $12 \mathrm{~cm}$, although they could be visualized smaller or bigger than their actual size in the simulation snapshots. Robots are able to measure their distances to the obstacles existing in the neighborhood or to the other neighboring robot. The neighborhood range is an adjustable parameter that can be modified in different tests. The virtual forces in section III were implemented in the robots in order to demonstrate the "diagonal line-up" behavior. In these test, the coefficient parameters of the method were set as following: $\eta=0.1, \lambda=0.1$, based on the dynamics of simulated robots to achieve a maximum speed of $0.1 \mathrm{~m} / \mathrm{s}, c_{1}=1$ and $\mu_{1}=\mu_{2}=\mu_{3}=1$ to treat all the forces with equal coefficients.

Fig. 19 shows the virtual forces that the robots generate in the "diagonal line-up" behavior in two configurations. Each arrow in a place shows the magnitude and the direction of virtual forces that would be applied to another robot if it was located in that place. By adding (or removing) robots to these scenarios the configuration of forces will change, however, these figures only show the virtual forces in the current setup of the figures before adding another robot. These forces are obtained by implementing the equations (12) to (15).

3) Validation: Fig. 20 shows a series of snapshots during a simulation that show the functionality of the "diagonal line" behavior and swarm's cross-wind movement and obstacle avoidance behavior. The first frame of this figure shows that 10 robots are released randomly in one part of the environment. The next frames demonstrate the "diagonal line-up" behavior, where they get apart from each other towards a diagonal line. The robots maintain the diagonal line formation and move 


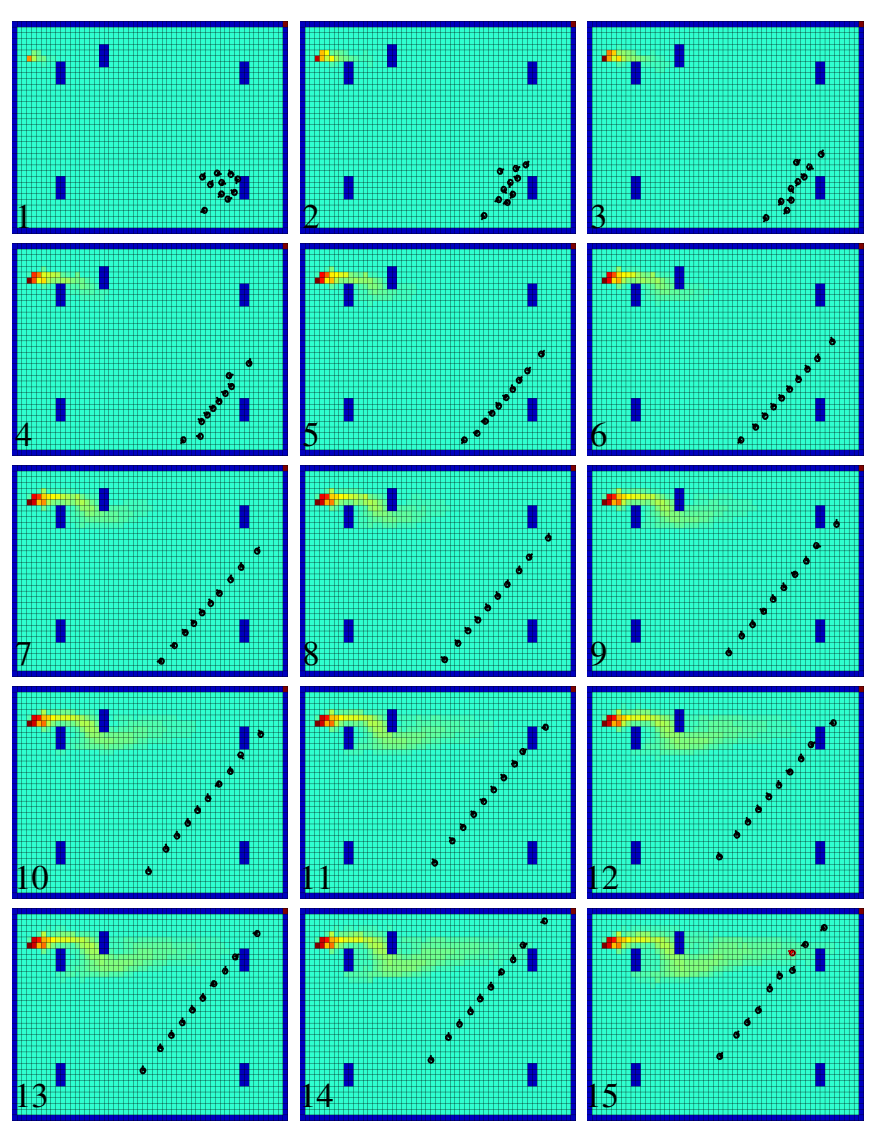

Fig. 20. Ten swarm robots searching in an environment for possible odor sources. In this scenario: $D_{x}=0.3, D_{y}=0.3, D_{r}=0.45, F s_{t h}=0.01$ and $\beta=0.1$.

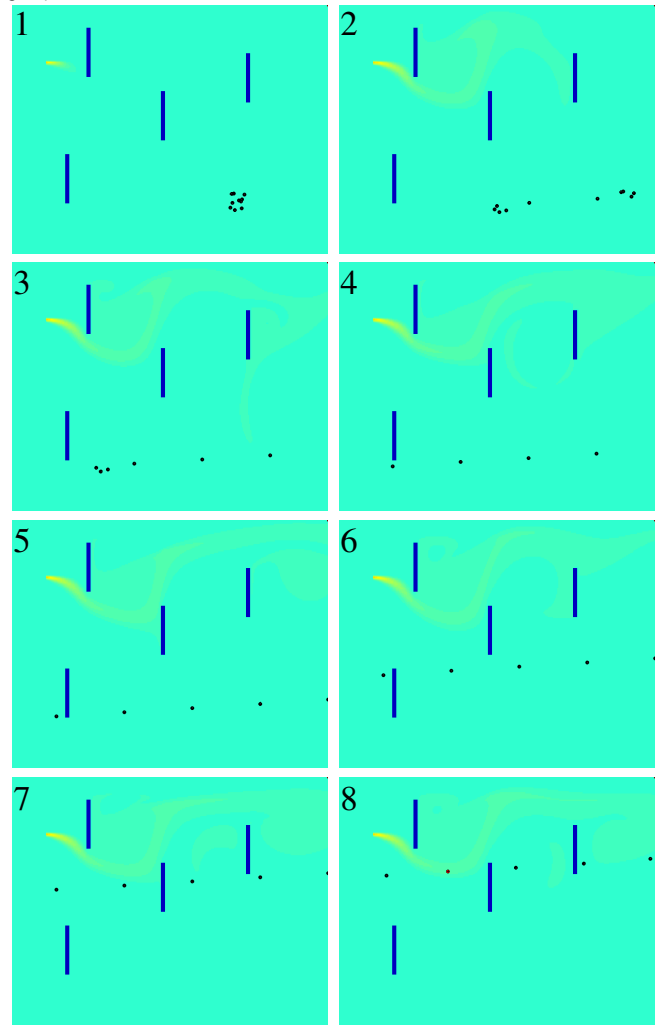

Fig. 21. Ten swarm robots searching in a large environment for possible odor sources. The swarm dynamically changes its topology to deal with environmental changes. The airflow is $0.5 \mathrm{~m} / \mathrm{s}$ from left to right. The robots perform the diagonal line-up behavior until one of them finds the plume.
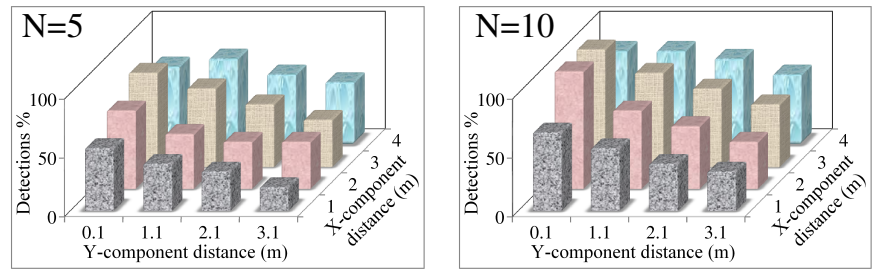

Fig. 22. Odor plume detection success of diagonal line-up behavior, during 15 tests using 5 (left) and 10 (right) swarm robots in each configuration.

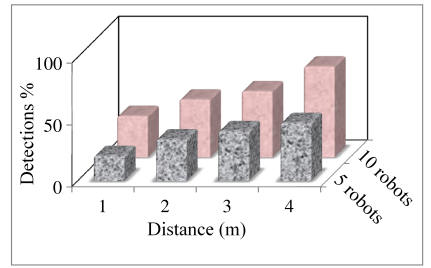

Fig. 23. Odor plume detection success of hyperbole behavior, during 15 tests using 5 and 10 swarm robots in each configuration.

towards cross-wind and avoid the obstacles. The last frame shows that a robot (the red one) gets into the plume and detects it. In this scenario $D_{x}$ and $D_{y}$ were set manually to $D_{x}=D_{y}=0.3 \mathrm{~m}$ and $D_{r}$ was set to $0.45 \mathrm{~m}$. In this test, $\Delta_{d}=1 \mathrm{~m}$, i.e. the range of communication between the robots is considered to be 1 meter.

4) Evaluation: The method was tested in a large environment $\left(30 \times 40 \mathrm{~m}^{2}\right)$ with 5 and 10 robots repeatedly. Fig. 21 shows a part of this environment that is $10 \times 15 \mathrm{~m}^{2}$ and includes an ethanol source. The release rate was set to $0.01 \mathrm{~g} / \mathrm{s}$ and the wind speed was $0.5 \mathrm{~m} / \mathrm{s}$. The robots perform the diagonal line-up behavior until one of them finds the plume. To evaluate the optimization results we measured the plume detection ability of swarm robots in two different formation strategies; 2D hyperbole formation and diagonal line formation strategy. In diagonal line formation, we manually set the parameter of distance between the robots $\left(D_{x}\right)$ to $1,2,3$ and 4 and $\left(D_{y}\right)$ to $0.1,1.1,2.1$ and 3.1 meters in different tests to find the best configuration. In $2 \mathrm{D}$ hyperbole formation the desired distance between neighboring robots was set to 1,2,3 and $4 m$ (similar to $D_{x}$ ). Each test was repeated for 15 times for every formation and value of $D_{x}$ and $D_{y}$. If at least one robot could detect the odor plume in less than one minute after the swarm formation was established, we consider a success in plume detection. The number of successfully detecting the odor plume was counted.

The results, in Fig. 22, show that the best performance between tested configurations is the one with diagonal line up formation when $D_{y}$ is $0.1 m$ and $D_{x}$ is $2 m$ or $3 \mathrm{~m}$. On the other hand, using the results of optimization in section II (Fig. 10 and equations (10), (11)), in the conditions of these simulations, the best formation strategy is diagonal line formation when $D_{y}$ is $0.17 \mathrm{~m}$ and $D_{x}$ is $2.52 \mathrm{~m}$. It should be mentioned that since there is no significant difference between the temperatures of various heights in our simulation arenas, the results of simulations are compared to the neutral environmental conditions (class A). The best configuration between the simulated ones are very close to the found analytic formation. This validates the optimization achievements. 


\section{B. Experimental Results}

In addition to simulations, the method was experimented with our currently available robotic facilities.

1) The robots: A set of $\mathrm{LSE}_{\mathrm{mini}}{ }^{2}$ robots were developed at our laboratory based on the $2 \mathrm{WD}$ miniQ ${ }^{3}$ platform. The motivation behind this was to build cheap and simple robotic units for swarm olfactory research.

The LSE miniQ (presented in Fig. 24) communicates with a host computer using XBee. ${ }^{4}$ The host computer runs ROS programs (nodes) to control the robots. Each robot is controlled by an individual ROS node. A single computer can run several ROS nodes, i.e., a swarm of LSE miniQs. Each robot contains an e2v MiCS-5524 gas sensor to allow the detection of volatile organic compounds. Two LEDs (one blue and one red) placed on the top of the robot are used for visual tracking by a camera mounted at the ceiling of the laboratory providing regular corrections to their odometry. The robots repeatedly broadcast their localization data, providing means to estimate the distance to their neighbors. In these tests, the airflow was intentionally ventilated and controlled towards the $\mathrm{X}$-axis of the robots and wind speed was manually provided (broadcasted) to the robots.

2) Realistic Environment: The method was tested in the reduced scale environment shown in Fig. 25. This arena, with $3 \times 4 \mathrm{~m}^{2}$ area by 0.5 meters height, has controlled ventilation through a manifold that extracts air from the testing environment through a honeycomb mesh integrated into one of the walls. The opposite surface of the environment contains a similar mesh that allows the entrance of clean air that flows through the environment. A controlled acetone gas source using bubblers is pumped to arbitrary places of the environment through a set of PolyVinyl Chloride (PVC) tubes. The acetone release rate was about $0.01 \mathrm{~g} / \mathrm{s}$ during the tests. The ceiling of this testbed is covered by a sheet of transparent Plexiglas to be visualized from the outside.

3) Validation: Similar to the presented simulations, for evaluating the optimization results, the experiments were done with manual values for $D_{y}$ and $D_{x}$ testing diagonal line formation and also hyperbole formation while moving towards cross-wind. The wind speed was about $0.7 \pm 0.1 \mathrm{~m} / \mathrm{s}$ and we tested the diagonal line formation strategy with different number of robots $(2,3$ and 4$)$ in various sets of parameters $\left(D_{x}\right.$ from 0.5 to $3.0 \mathrm{~m}$ and $D_{y} 0.1 \mathrm{~m}$ and $0.5 \mathrm{~m}$ ). In different tests, one, two, three and four robots were released in one corner of the testbed and each test was repeated seven times and the plume detections were counted. We considered the following two positions for the odor source release: $(0,0.8)$ and $(2,2)$. Each particular test was repeated two times (once having the source at $(0,0.8)$ and another time at $(2,2))$. In each test if at least one of the robots detects the odor plume we consider a success in plume detection. Fig. 26 shows a series of snapshots in one of these experiments. Fig. 27 presents the trajectory of four robots in one experiment and Fig. 28 shows the chemical map of the environment generated by the data captured by the

\footnotetext{
${ }^{2} \mathrm{http}: / /$ lse.isr.uc.pt/news/lseminiqrobot

${ }^{3}$ http://www.dfrobot.com

${ }^{4}$ http://www.digi.com/xbee/
}

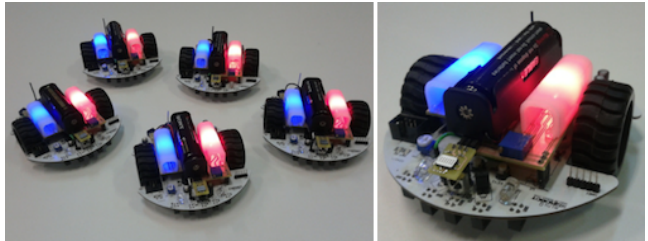

Fig. 24. The developed LSE MiniQ robots containing gas sensors, XBee modules and LEDs.
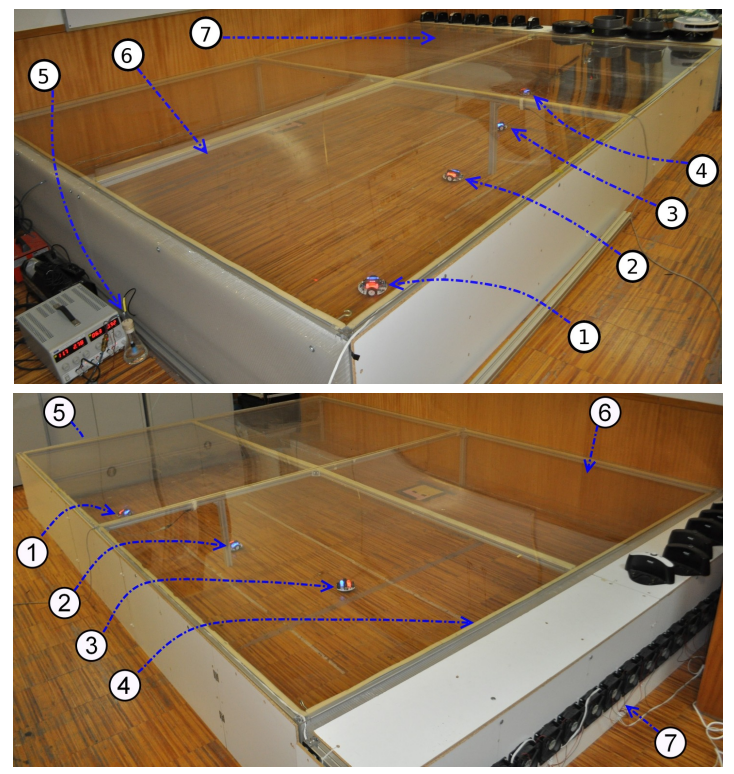

Fig. 25. The realistic testbed environment viewing from two different angles. 1,2,3,4: robots, 5: odor source (Acetone release bubbler), 6: transparent Plexiglas ceiling, 7: ventilation system.

robots after an interpolation process.

Fig. 29 and 30 show the results of the realistic experiments, testing hyperbole and diagonal line formations with different parameters. Comparing the results shown Fig. 29 to the three charts in Fig. 30 shows that hyperbole formation provides less detections in comparison to diagonal line formation. The results in Fig. 30 show that if there are four robots in this arena, when the X-distance between each pair of robots is one meter, they can detect the odor sources in 100 percent of the tests. It should be mentioned that we can not increase $D_{x}$ more than one meter (for four robots) since the length of the testbed is $4 \mathrm{~m}$. Fig. 30 (middle) shows that if there are three robots in this environment they reach to one hundred percent detection when their X-distance is $1.5 \mathrm{~m}$. The results in Fig. 30 (down) show that if there are two robots, they detect the odor patches when their X-distance is $2.5 \mathrm{~m}$. Since two robots can provide (almost a) full coverage over this area, it is obvious that having more number of robots also will fully cover the environment and will not provide useful information regarding optimal distance between the robots. Therefore, considering the small size of the testbed, the best distance of two robots should be considered to be the best distance of the robots in this environment. The best $\mathrm{X}$-component distance of two robots is $2.5 \mathrm{~m}$ and the Y-component can be both 0.1 and $0.5 \mathrm{~m}$. During these tests, changing $D_{x}$ from 0.1 to $0.5 \mathrm{~m}$ 


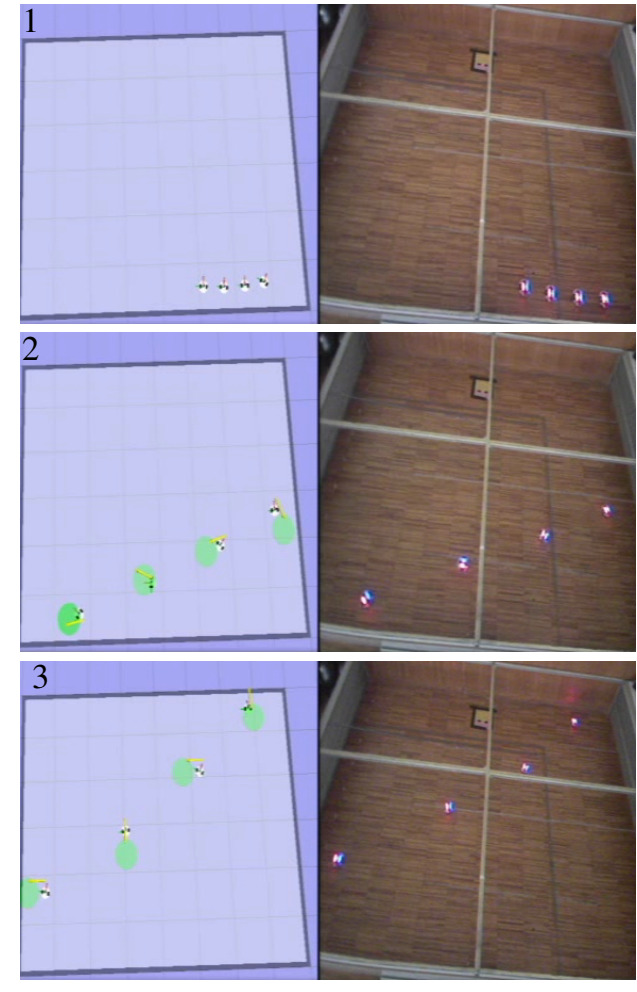

Fig. 26. A series of snapshots during an experiment while $N=4, D_{y}=$ $0.5 \mathrm{~m}$ and $D_{x}=1 \mathrm{~m}$. The pictures in the left of each frame shows the ROS rviz framework and the right pictures show the real robots in the testbed.
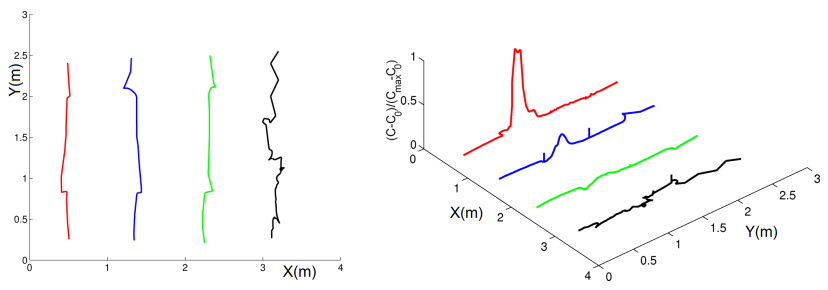

Fig. 27. Left: the trajectory of the robots during an experiment while $N=4$, $D_{y}=0.1 \mathrm{~m}$ and $D_{x}=0.75 \mathrm{~m}$. Right: the Z-axis in this figure shows the chemical concentration sensed by the robots during their maneuvers.

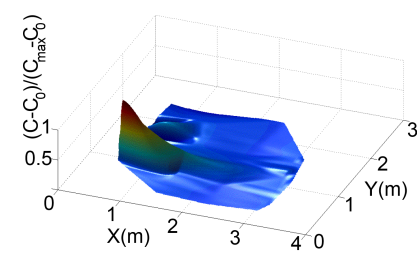

Fig. 28. The chemical map of the environment generated by the robots after the experiment described in Fig. 27.

does not show a significant change in the results since the testbed is small.

Using equations (10) and (11), the optimal X-distance in this configuration is $2.1 \mathrm{~m}$ and the optimal Y-distance is $0.15 \mathrm{~m}$ that approximately agree with the results of the real experiments. The difference between the results of the real world experiments and the results of the optimizations is because the airflow and the detection of the sensors in reality are not as constant as what is assumed in the analytical optimizations. Nevertheless, the realistic experiments show

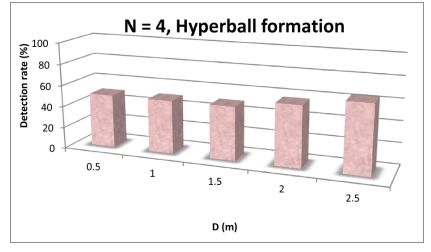

Fig. 29. Detection rate in hyperbole formation against different values for the distance between the neighboring robots (D).

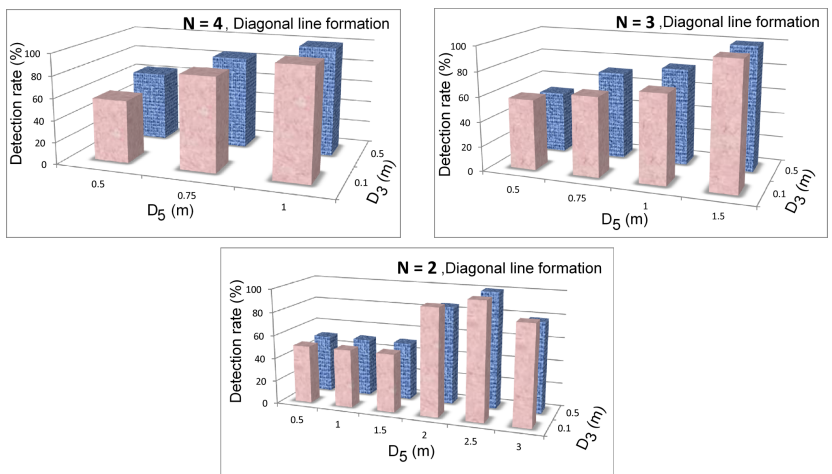

Fig. 30. Detection rate of diagonal line formation against different values for $D_{y}$ and $D_{x}$, when the number of robots is 4,3 , and 2 .

very close results to the optimizations.

4) Experiments in indoor environments: To study the functionality of the method in real-world indoor environments, experiments were carried out in the second floor of the Institute of Systems and Robotics (ISR) in the University of Coimbra. Two cameras were mounted in the fourth floor of this building that observe an area of $5 \times 13 \mathrm{~m}^{2}$ of the testing area. Acetone was released using a bubbler and a set of fans were used to generate airflow. The average of the wind speed in different points of this area was about $0.8 \mathrm{~m} / \mathrm{s}$. Fig. 31 shows a picture of one of these tests.

Fig. 32.(left) is an example that shows the trajectory of four robots performing diagonal line-up behavior when $D_{x}$ was set to $1.5 \mathrm{~m}$ and $D_{y}$ to $0.1 \mathrm{~m}$. Fig. 32.(right) shows the chemical concentration sensed by the robots during their trajectory. Fig. 33 shows the chemical map of this environment generated from the data captured by the robots after an interpolation process. These results are very similar to the experiments in the controlled environment in section IV-B3.

In these experiments we qualitatively observed that the "diagonal-line" topology provides higher success rate in plume finding than the hyperbole formation. However, we were not able to quantitatively evaluate the performance of each topology in this setup due to inconsistency in the obtained results. The main difference between these experiments and the previous ones was the state of the air-flow. When all of the fans are set to their maximum power, the airflow speed measured by an anemometer at the position of the odor source was about $1.2 \pm 0.2 \mathrm{~m} / \mathrm{s}$, but at the same time, 8 meters away from the source (towards downwind direction) the airflow speed was about $0.6 \pm 0.2 \mathrm{~m} / \mathrm{s}$. Since the airflow in this setup is not constant in various locations of the working area, we are not able to have a comparison between these experiments 


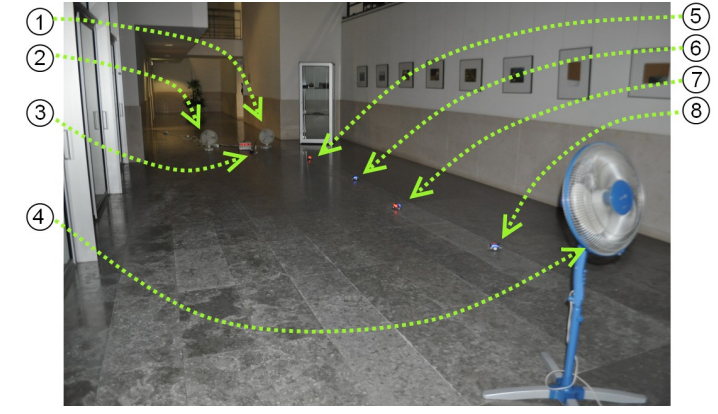

Fig. 31. Four robots performing diagonal-lineup behavior in an indoor environment. Fans: 1, 2, 4. Acetone bubbler: 3. LSE-MiniQ robots: 5, 6, 7,8 .
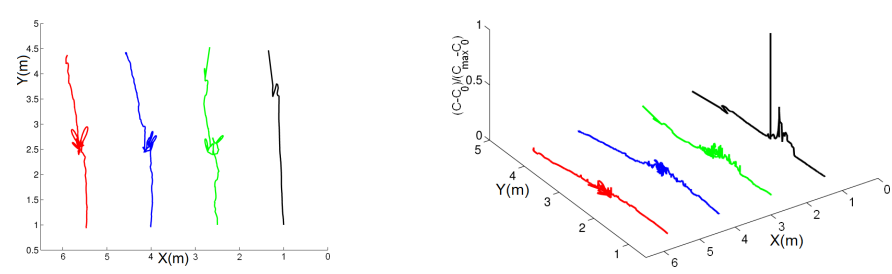

Fig. 32. Left: Trajectory of the robots in one experiment while $D_{x}=1.5 \mathrm{~m}$ and $D_{y}=0.1 \mathrm{~m}$. Right: The chemical data sensed by the robots during their path. In this experiment the odor source was located at $(0,2) \mathrm{m}$.

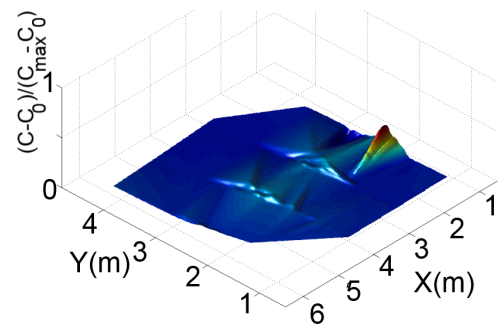

Fig. 33. The chemical map of the environment generated by the robots, corresponding to the trajectories in Fig. 32.

and the optimizations in section II. These experiments validate the functionality of the wind-biased virtual forces in the real scenarios. Still, for evaluating the found optimal configurations in real world, outdoor large scale tests are required.

\section{CONCLUSIONS AND FUTURE WORKS}

Considering cross-wind movement for a swarm we studied the optimal formation of swarm robots that maximizes their probability of finding an odor plume in an unknown environment. The formation of the optimal solutions is diagonal line configuration, with equal distance between each pair of neighboring sensors. The distance between neighboring pairs in optimal configurations depends mainly on the wind speed and the environmental conditions, whereas the number of sensors and the swarm's crosswind movement distance do not show significant impact on optimal configurations. Mathematical functions that can accurately estimate the optimal distances based on the wind speed were computed by nonlinear regression estimation. Moreover, swarm robotics wind-biased attractive/repulsive virtual forces were designed to emerge to the optimal configurations. The method was tested and validated in simulations and in a reduced scale realistic environment under laminar and stable controlled airflow. The results verify the functionality of the swarming formation strategy and also validate the obtained optimization results.

Finally, there are some future works that should be discussed here. We presented analytical optimizations for odor plume finding problem and validated them through simple simulation and realistic experiments. However, outdoor experiments are still required to evaluate the conclusions of this study in the real-word. This is the main future work of this study that can be done in a large scale urban environment. The other point is that although the swarm control strategy presented in this paper is adaptive to the changes in the wind direction and speed (due to equation (10), (11) and (12)), during the optimization process we have assumed that the wind state is stable. However, if the airflow direction is very unstable (such that the odor distribution models do not hold) the analytical optimizations of this paper will not be valid. Studying this problem under very unstable conditions can provide significant contributions for the community. Last but not the least, studying the stability of wind biased attraction/repulsion method considering various wind profiles in different environments is another future work of this paper.

\section{REFERENCES}

[1] M. Dunbabin and L. Marques, "Robotics for environmental monitoring: Significant advancements and applications," IEEE Robotics and Automation Magazine, vol. 19, no. 1, March 2012.

[2] R. Russell, D. Thiel, R. Deveza, and A. Mackay-Sim, "A robotic system to locate hazardous chemical leaks," in Proc. IEEE Int. Conf. on Robotics and Automation, 1995.

[3] H.-L. Fu, H.-C. Chen, and P. Lin, "APS: Distributed air pollution sensing system on Wireless Sensor and Robot Networks," Computer Communications, vol. 35, no. 9, pp. 1141 - 1150, 2012.

[4] V. Hernandez Bennetts, A. Lilienthal, P. Neumann, and M. Trincavelli, "Mobile robots for localizing gas emission sources on landfill sites: is bio-inspiration the way to go?" Frontiers in Neuroengineering, vol. 4, p. 20, 2012.

[5] H. Wang, M. Zhang, J. Wang, and M. Huang, "Engineering an emergency search and rescue application with wireless sensor network and mobile robot," in Int. Conf. on Measuring Technology and Mechatronics Automation, vol. 2, 2010, pp. 112-115.

[6] P. Roberts and D. Webster, Turbulent diffusion. ASCE Press, Reston, Virginia, 2002.

[7] J. Crimaldi, M. Wiley, and J. Koseff, "The relationship between mean and instantaneous structure in turbulent passive scalar plumes," Journal of Turbulence, vol. 3, no. 14, pp. 1-24, 2002.

[8] O. Sutton, "The problem of diffusion in the lower atmosphere," Quarterly Journal of the Royal Meteorological Society, vol. 73, no. 317318, pp. 257-281, 1947.

[9] F. Gifford, "Peak to average concentration ratios according to a fluctuating plume dispersion model." International journal of air pollution, vol. 3, p. 253, 1960.

[10] E. Balkovsky and B. Shraiman, "Olfactory search at high Reynolds number," in Proc. National Academy of Science, 99(20), USA, 2002.

[11] R. Russell, A. Bab-Hadiashar, R. Shepherd, and G. Wallace, "A comparison of reactive robot chemotaxis algorithms," Robotics and Autonomous Systems, vol. 45, no. 2, pp. 83-97, 2003.

[12] G. Cabrita and L. Marques, "Divergence-based odor source declaration," in Control Conference (ASCC), 2013 9th Asian. IEEE, 2013.

[13] G. Kowadlo and R. A. Russell, "Robot odor localization: a taxonomy and survey," The Int. Journal of Robotics Research, vol. 27, no. 8, pp. 869-894, 2008.

[14] H. Ishida, Y. Wada, and H. Matsukura, "Chemical sensing in robotic applications: A review,” IEEE Sensors Journal, vol. 12, no. 11, pp. 31633173,2012 
[15] A. Marjovi and L. Marques, "Swarm robotic plume tracking for intermittent and time-variant odor dispersion," in European Conf. on Mobile Robotics, Barcelona, Spain, 2013.

[16] W. Li, J. Farrell, and R. Cardé, "Tracking of fluid-advected odor plumes: strategies inspired by insect orientation to pheromone," Adaptive Behavior, vol. 9, no. 3-4, p. 143, 2001

[17] T. Lochmatter and A. Martinoli, "Tracking odor plumes in a laminar wind field with bio-inspired algorithms," in Experimental Robotics. Springer, 2009.

[18] H. Ishida, G. Nakayama, T. Nakamoto, and T. Moriizumi, "Controlling a gas/odor plume-tracking robot based on transient responses of gas sensors," IEEE Sensors Journal, vol. 5, no. 3, pp. 537-545, 2005.

[19] L. Marques and A. de Almeida, "ThermalSkin: a distributed sensor for anemotaxis robot navigation," in Proc. 5th IEEE Int. Conf. on Sensors, South Korea, 2006.

[20] J. A. Farrell, S. Pang, and W. Li, "Plume mapping via hidden markov methods," IEEE Trans. on Systems, Man, and Cybernetics, Part B: Cybernetics, vol. 33, no. 6, pp. 850-863, 2003.

[21] A. Marjovi and L. Marques, "Multi-robot odor distribution mapping in realistic time-variant conditions," in Proc. IEEE Int. Conf. on Robotics and Automation, Hong Kong, China, 2014.

[22] A. Lilienthal and T. Duckett, "Building gas concentration gridmaps with a mobile robot," Robotics and Autonomous Systems, vol. 48, no. 1, pp. 3-16, 2004

[23] S. Pang and J. A. Farrell, "Chemical plume source localization," IEEE Trans. on Systems, Man, and Cybernetics, Part B: Cybernetics, vol. 36, no. 5, pp. 1068-1080, 2006.

[24] A. Marjovi and L. Marques, "Multi-robot olfactory search in structured environments," Robotics and Autonomous Systems, vol. 52, no. 11, pp. 867-881, 2011.

[25] T. Lochmatter, E. Aydin, I. Navarro, and A. Martinoli, "A plume tracking algorithm based on crosswind formations," in Proc. Int. Symp. on Distributed Autonomous Robotics Systems, Lausanne, Switzerland, 2010.

[26] X. Cui, C. Hardin, R. Ragade, and A. Elmaghraby, "A swarm approach for emission sources localization," in IEEE Int. conf. on Tools with Artificial Intelligence, FL, USA, 2004.

[27] L. Marques, U. Nunes, and A. De Almeida, "Odour searching with autonomous mobile robots: An evolutionary-based approach," Proc. IEEE Int. Conf. on Advanced Robotics, pp. 494-500, 2003.

[28] F. Li, Q. Meng, S. Bai, J. Li, and D. Popescu, "Probability-PSO algorithm for multi-robot based odor source localization in ventilated indoor environments," Intelligent Robotics and Applications, 2008.

[29] L. Marques, U. Nunes, and A. de Almeida, "Particle swarm-based olfactory guided search," Autonomous Robots, vol. 20, no. 3, pp. 277$287,2006$.

[30] M. Turduev, G. Cabrita, M. Kırtay, V. Gazi, and L. Marques, "Experimental studies on chemical concentration map building by a multi-robot system using bio-inspired algorithms," Autonomous Agents and Multi-Agent Systems, vol. 28, no. 1, pp. 72-100, 2014.

[31] K. Krishnanand and D. Ghose, "Theoretical foundations for rendezvous of glowworm-inspired agent swarms at multiple locations," Robotics and Autonomous Systems, vol. 56, no. 7, pp. 549-569, 2008.

[32] A. Hayes, A. Martinoli, and R. Goodman, "Distributed odor source localization," IEEE Sensors Journal, vol. 2, no. 3, pp. 260-271, 2002.

[33] D. Zarzhitsky, D. Spears, and W. Spears, "Swarms for chemical plume tracing," in Proc. of the Swarm Intelligence Symposium, USA, 2005.

[34] A. Marjovi and L. Marques, "Multi-robot topological exploration using olfactory cues," in Distributed Autonomous Robotic Systems, ser. Springer Tracts in Advanced Robotics, 2013, vol. 83, pp. 47-60.

[35] L. Marques, U. Nunes, and A. Almeida, "Olfaction-based mobile robot navigation," Thin Solid Films, vol. 418, no. 1, pp. 51-58, 2002.

[36] Z. Pasternak, F. Bartumeus, and F. Grasso, "Lévy-taxis: a novel search strategy for finding odor plumes in turbulent flow-dominated environments," Journal of Physics A: Mathematical and Theoretical, vol. 42, p. 434010, 2009.

[37] G. Ferri, E. Caselli, V. Mattoli, A. Mondini, B. Mazzolai, and P. Dario, "SPIRAL: A novel biologically-inspired algorithm for gas/odor source localization in an indoor environment with no strong airflow," Robotics and Autonomous Systems, vol. 57, no. 4, pp. 393-402, 2009.

[38] M. Sabelis and P. Schippers, "Variable wind directions and anemotactic strategies of searching for an odour plume," Oecologia, vol. 63, no. 2, pp. 225-228, 1984.

[39] D. Hamel, M. Chwastek, B. Farouk, K. Dandekar, and M. Kam, "A computational fluid dynamics approach for optimization of a sensor network," in Proc. of the IEEE Int. Workshop on Measurement Systems for Homeland Security, Contraband Detection and Personal Safety, 2006, pp. 38-42.

[40] J. Hayes, S. Beirne, K.-T. Lau, and D. Diamond, "Evaluation of a low cost wireless chemical sensor network for environmental monitoring," in IEEE Sensors Journal, 2008, pp. 530-533.

[41] A. Marjovi and L. Marques, "Optimal spatial formation of swarm robotic gas sensors in odor plume finding," Autonomous Robots, vol. 35, pp. 93109,2013

[42] C. Jones, "On the structure of instantaneous plumes in the atmosphere," Journal of Hazardous Materials, vol. 7, no. 2, pp. 87-112, 1983.

[43] F. Gifford, "Turbulent diffusion-typing schemes: a review," Nuclear Safety, vol. 17, no. 1, pp. 68-86, 1976.

[44] G. Briggs, "Diffusion estimation for small emissions ATDL, contribution file No. 97," Air Resources Atmospheric Turbulence and Diffusion Laboratory, NOAA, Oak Ridge, Tennessee, 1973.

[45] K. Arshak, E. Moore, G. Lyons, J. Harris, and S. Clifford, "A review of gas sensors employed in electronic nose applications," Sensor Review, vol. 24, no. 2, pp. 181-198, 2004.

[46] Q. Meng, W. Yang, Y. Wang, and M. Zeng, "Collective odor source estimation and search in time-variant airflow environments using mobile robots," Sensors, vol. 11, no. 11, pp. 10415-10443, 2011.

[47] K. Cheng, V. Acevedo-Bolton, R. Jiang, N. Klepeis, W. Ott, O. Fringer, and L. Hildemann, "Modeling exposure close to air pollution sources in naturally ventilated residences: Association of turbulent diffusion coefficient with air change rate," Environmental science \& technology, 2011.

[48] W. Press, B. Flannery, S. Teukolsky, and W. Vetterling, "Direction set (Powell's) methods in multidimensions," Numerical recipes in $\mathrm{C}++$ : The art of scientific computing, vol. 1992, pp. 417-424, 2002.

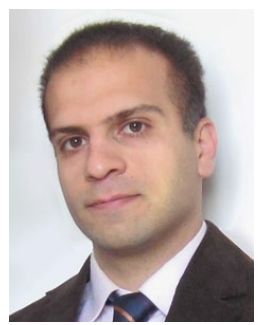

Ali Marjovi has a Ph.D. degree in Electrical and Computer Engineering from University of Coimbra, Portugal in the field of Distributed Olfactory Robotics. He received his M.Sc. degree in Computer Engineering from Sharif University of Technology and his B.Eng. degree in the same field from the University of Isfahan in Iran. He is currently a postdoc researcher at the Distributed Intelligent Systems and Algorithms Laboratory, EPFL, Switzerland. His research interests include multi-robot and swarm robotics, olfactory robotics, and intelligent vehicles.

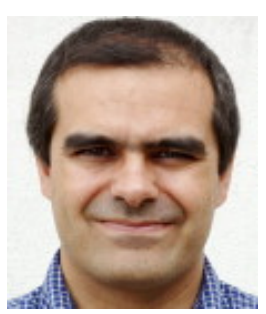

Lino Marques has a Ph.D. degree in Electrical Engineering from the University of Coimbra, Portugal. He is currently a Senior Lecturer at the Department of Electrical and Computer Engineering, University of Coimbra, and he heads the Field and Service Robotics of the Institute for Systems and Robotics from that university (ISR-UC). His main research interests include smart sensors and robotics perception, mechatronics, and field robotics. 